\title{
Article
}

\section{The Older Finnish Twin Cohort - 45 Years of Follow-up}

\author{
Jaakko Kaprio ${ }^{1,2}$, Sailalitha Bollepalli ${ }^{1}$, Jadwiga Buchwald ${ }^{1}$, Paula Iso-Markku ${ }^{1,3}$, Tellervo Korhonen ${ }^{1}$, Vuokko Kovanen ${ }^{4}$, \\ Urho Kujala ${ }^{5}$, Eija K. Laakkonen ${ }^{4}$, Antti Latvala ${ }^{1}$, Tuija Leskinen ${ }^{6}$, Noora Lindgren ${ }^{7}$, Miina Ollikainen ${ }^{1}$, Maarit Piirtola ${ }^{1}$, \\ Taina Rantanen ${ }^{4}$, Juha Rinne ${ }^{7,8}$, Richard J. Rose ${ }^{9}$, Elina Sillanpää ${ }^{4}$, Karri Silventoinen ${ }^{2,10}$, Sarianna Sipilä ${ }^{4}$, \\ Anne Viljanen ${ }^{4}$, Eero Vuoksimaa ${ }^{1}$ and Katja Waller ${ }^{5}$ \\ ${ }^{1}$ Institute for Molecular Medicine FIMM, University of Helsinki, Helsinki, Finland, ${ }^{2}$ Department of Public Health, University of Helsinki, Helsinki, Finland, \\ ${ }^{3}$ Department of Clinical Physiology and Nuclear Medicine, HUS Medical Imaging Center, Helsinki University Central Hospital and University of Helsinki, Helsinki, \\ Finland, ${ }^{4}$ Gerontology Research Center and Faculty of Sport and Health Sciences, University of Jyväskylä, Jyväskylä, Finland, ${ }^{5}$ Faculty of Sport and Health \\ Sciences, University of Jyväskylä, Jyväskylä, Finland, ${ }^{6}$ Department of Public Health, University of Turku and Turku University Hospital, Turku, Finland, ${ }^{7}$ Turku \\ PET Centre, University of Turku, Turku, Finland, ${ }^{8}$ Division of Clinical Neurosciences, Turku University Hospital, Turku, Finland, ${ }^{9}$ Department of Psychological and \\ Brain Sciences, Indiana University, Bloomington, IN, USA and ${ }^{10}$ Population Research Unit, Faculty of Social Sciences, University of Helsinki, Helsinki, Finland
}

\begin{abstract}
The older Finnish Twin Cohort (FTC) was established in 1974. The baseline survey was in 1975, with two follow-up health surveys in 1981 and 1990. The fourth wave of assessments was done in three parts, with a questionnaire study of twins born during 1945-1957 in 2011-2012, while older twins were interviewed and screened for dementia in two time periods, between 1999 and 2007 for twins born before 1938 and between 2013 and 2017 for twins born in 1938-1944. The content of these wave 4 assessments is described and some initial results are described. In addition, we have invited twin-pairs, based on response to the cohortwide surveys, to participate in detailed in-person studies; these are described briefly together with key results. We also review other projects based on the older FTC and provide information on the biobanking of biosamples and related phenotypes.
\end{abstract}

Keywords: Aging; alcohol; cohort study; dementia; epigenetics; hypertension; longitudinal; physical activity; review; smoking; twins

(Received 29 June 2019; accepted 17 July 2019)

The older Finnish Twin Cohort (FTC) was established 45 years ago and has been described extensively in prior reviews (Kaprio, 2006, 2013; Kaprio \& Koskenvuo, 2002). Here, we will review the continued follow-up of the cohort, describe important legislative changes that affect how the cohort can and will be available to researchers and describe in more detail some of the more extensive research projects based partially or entirely on the adult cohort.

Since its establishment, the FTC has been hosted by the University of Helsinki and the primary goal in the 1970s and early 1980s was to study twin-pairs discordant for smoking, as the causal evidence for smoking in major diseases was still lacking. During the 1980s, quantitative genetic modeling became more common in published papers based on the cohort, primarily through collaborations with Indiana University and Professor Richard J. Rose. In the 1990s, as a result of collaboration with the National Public Health Institute of Finland and Professors Leena Peltonen and Jussi Huttunen, DNA samples were collected with the aim of addressing the genetic architecture of complex traits and diseases

Author for correspondence: Jaakko Kaprio, Email: jaakko.kaprio@helsinki.fi

Cite this article: Kaprio J, Bollepalli S, Buchwald J, Iso-Markku P, Korhonen T, Kovanen V, Kujala U, Laakkonen EK, Latvala A, Leskinen T, Lindgren N, Ollikainen M, Piirtola M, Rantanen T, Rinne J, Rose RJ, Sillanpää E, Silventoinen K, Sipilä S, Viljanen A, Vuoksimaa E, and Waller K. (2019) The Older Finnish Twin Cohort - 45 Years of Follow-up. Twin Research and Human Genetics 22: 240-254, https://doi.org/10.1017/thg.2019.54 such as hypertension, obesity, migraine, diabetes and osteoarthritis, to mention a few of the first projects. We also expanded collaborations with other research groups in Finland, permitting much more detailed phenotyping and analyses in selected subsets of twins, some of which are described later. International collaborations have allowed for fruitful exchange of ideas, pooling of results and training of young scientists in multicenter studies.

\section{Data Collections}

\section{Questionnaire and Interview Studies}

Earlier questionnaires were mailed in 1975 and 1981 to all twins born before 1958 and living in Finland, identified from the population register of Finland; the 1990 third-wave questionnaire was targeted to twins born in 1930-1957 who had responded to either earlier questionnaire. Numbers and details are shown in the first three rows of Table 1 .

The fourth wave of cohortwide data collection has been conducted in three separate phases and with separate data collection methods. We invited the participation of twins based on their birth years (those born before 1938, 1938-1944 and 1945-1957) for studies conducted between 1999 and 2017. In addition, selected subsets of twins have been approached for more intensive clinical studies.

(c) The Author(s) 2019. This is an Open Access article, distributed under the terms of the Creative Commons Attribution licence (http://creativecommons.org/licenses/by/4.0/), which permits unrestricted re-use, distribution, and reproduction in any medium, provided the original work is properly cited. 
Table 1. Questionnaires and interviews of the adult twin cohort in four waves of data collection. The fourth wave was spread over two decades and used either interviews or questionnaires for data collection in three separate substudies defined by birth year

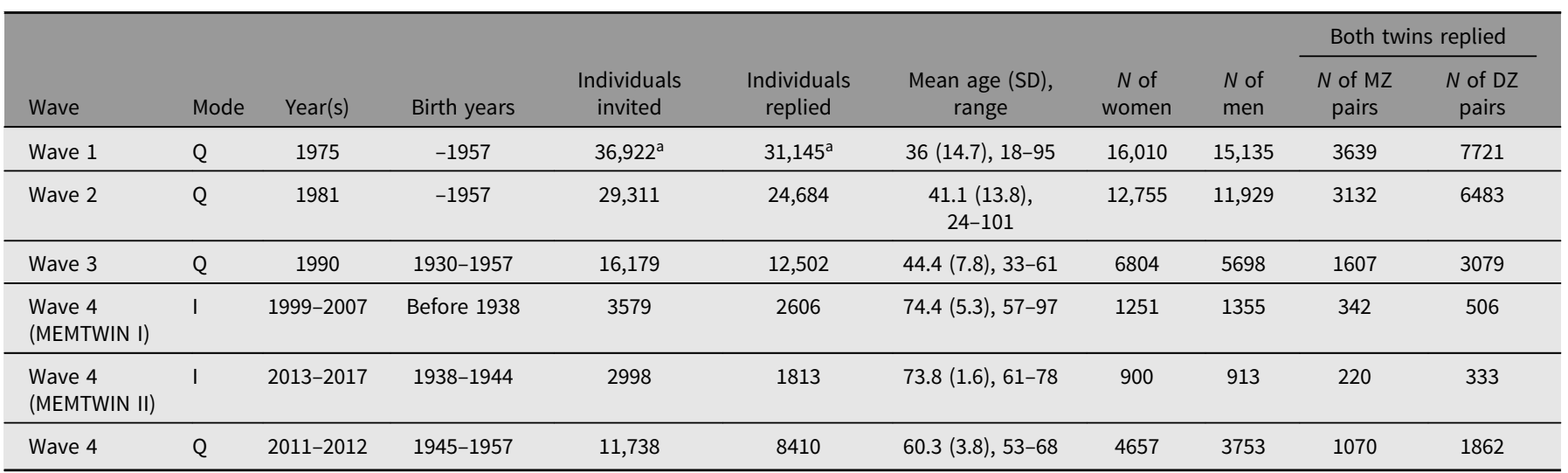

Note: I = Interview, $Q=$ Questionnaires

${ }^{a}$ At wave 1 , these numbers include nonbiological twins who satisfied the original selection criteria (pairs of persons with the same surname at birth, same birth date, same local community of birth and same sex) in 1974

\section{Cognitive Screening Studies}

Starting in 1999, twins from the FTC over 65 years old, and eventually including all those born before 1938, were invited to participate in a telephone interview assessing cognitive functioning. Two validated instruments were used: a telephone assessment of dementia (TELE; Gatz et al., 2002) and Telephone Interview for Cognitive Status (TICS; Brandt et al., 1988). Also, a 1-min category (animals) fluency task was administered. The first part of the data collection took place in 1999-2007 and included twins born before 1938. More specifically, monozygotic (MZ) twin-pairs (both members of a pair alive) were interviewed in 1999-2001 and dizygotic (DZ) twins and those with uncertain zygosity, irrespective of the co-twin's vital status, were interviewed in 2003-2007. All participants were also asked to provide a blood sample for extracting DNA. There were a total of 2606 twins who participated in the telephone interview at a mean age of 74 years. The study collection is termed MEMTWIN I in Table 1.

The second part of the data collection was performed in 20132017 and included cohorts born in 1938-1944 (MEMTWIN II in Table 1). All twins - regardless of co-twin's vital status - were asked to participate. Cognitive measures were similar to earlier data collection, with the exception of using the modified version of the TICS (TICS-m, including a delayed free recall of 10-word list). In addition to cognitive instruments, the Center for Epidemiological Studies Depression scale (CES-D) was also included (Radloff, 1977). All individuals were also asked to participate in a saliva sample collection for extraction of DNA. A total of 1813 twins participated in the telephone interview at a mean age of 74 years. Twins born in 1940-1944 were invited to take part in objective measures of physical activity. See below for details and first results.

These two data collections, collectively called MEMTWIN, have cognitive data available from a total of 4429 twins with a participation rate of $67 \%$ (Lindgren et al., 2019). Of these, 3614 have genotype data. Informed consent was obtained from participants, and the study protocol was approved by the Ethics Committee of the Hospital District of Southwest Finland. We are currently planning to administer postal questionnaires and telephone interviews for all FTC twins who reach 90 years of age. Selected pairs potentially discordant for cognitive impairment were invited to the national PET Centre, University of Turku for neuropsychological (NP) assessments, magnetic resonance imaging (MRI) and positron emission tomography (PET) imaging.

\section{Lifestyle, Health and Behavioral Changes Over 35 Years}

In the 2013 review (Kaprio, 2013), the mailed survey conducted among twins born in 1945-1957 was described in detail. In brief, the questionnaire had 85 questions (and 240 items). The topics covered items about contact with their co-twin, health and risk factors (blood pressure [BP] and cholesterol measurements, diabetes, breathlessness, back pain, bruxism, chronic diseases and use of common medications). Women were asked about hormone replacement therapy (HRT) use. Six questions on sleep were included. Eight items on work and pension intentions were asked next. Twelve questions asked a detailed smoking history, including two nicotine dependence scales, while seven questions were directed to alcohol quantity, frequency and problem drinking. Six items asked about leisure-time physical activity (LTPA) and sedentary behavior. Life events and traumatic experiences in childhood and adulthood were questioned, as well as items on social interactions. We repeated the four-item life satisfaction scale, and the brief assessment of extraversion and neuroticism (19 items together) asked in previous surveys. We included the CES-D depression scale. Finally, we asked about their current height and weight, as well as weight from 1 year and 5 years earlier, as well as their weight at age 50 and largest weight ever. Finally, the participants were asked to self-measure their waist circumference with a tape measure included with the questionnaire. We found that this had acceptably high validity when measured by health professionals in the Epigenetics of Hypertension study (Tuomela et al., in press). This has enabled longitudinal analyses spanning up to four measurements and 35 years of follow-up (see below for more detail on analyses related to alcohol, weight and physical inactivity). The details of the three subsets of wave 4 are also shown in Table 1, while Figure 1 gives the numbers of twins who have answered various combinations of surveys, which helps when planning longitudinal analyses. 
Panel A

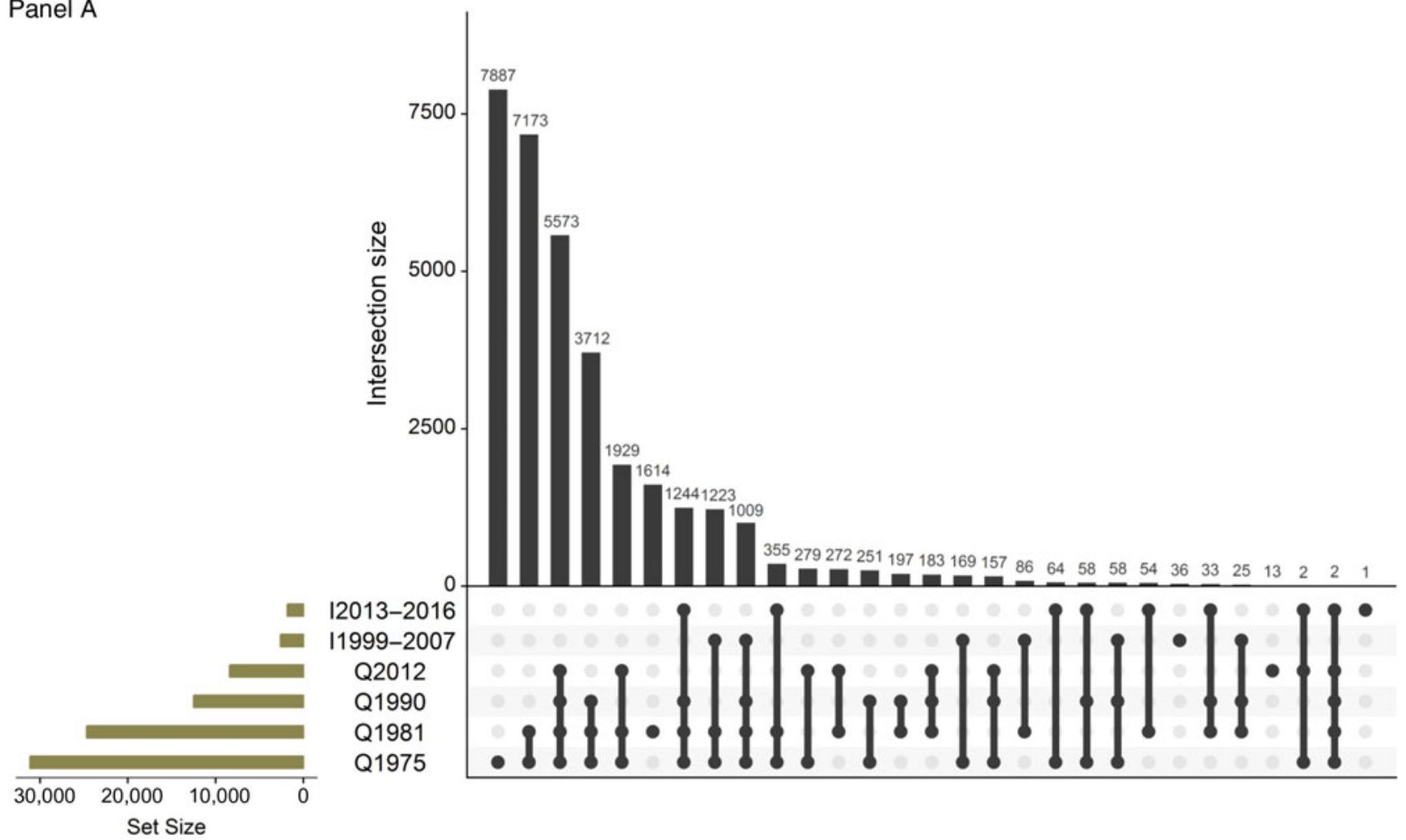

Panel B

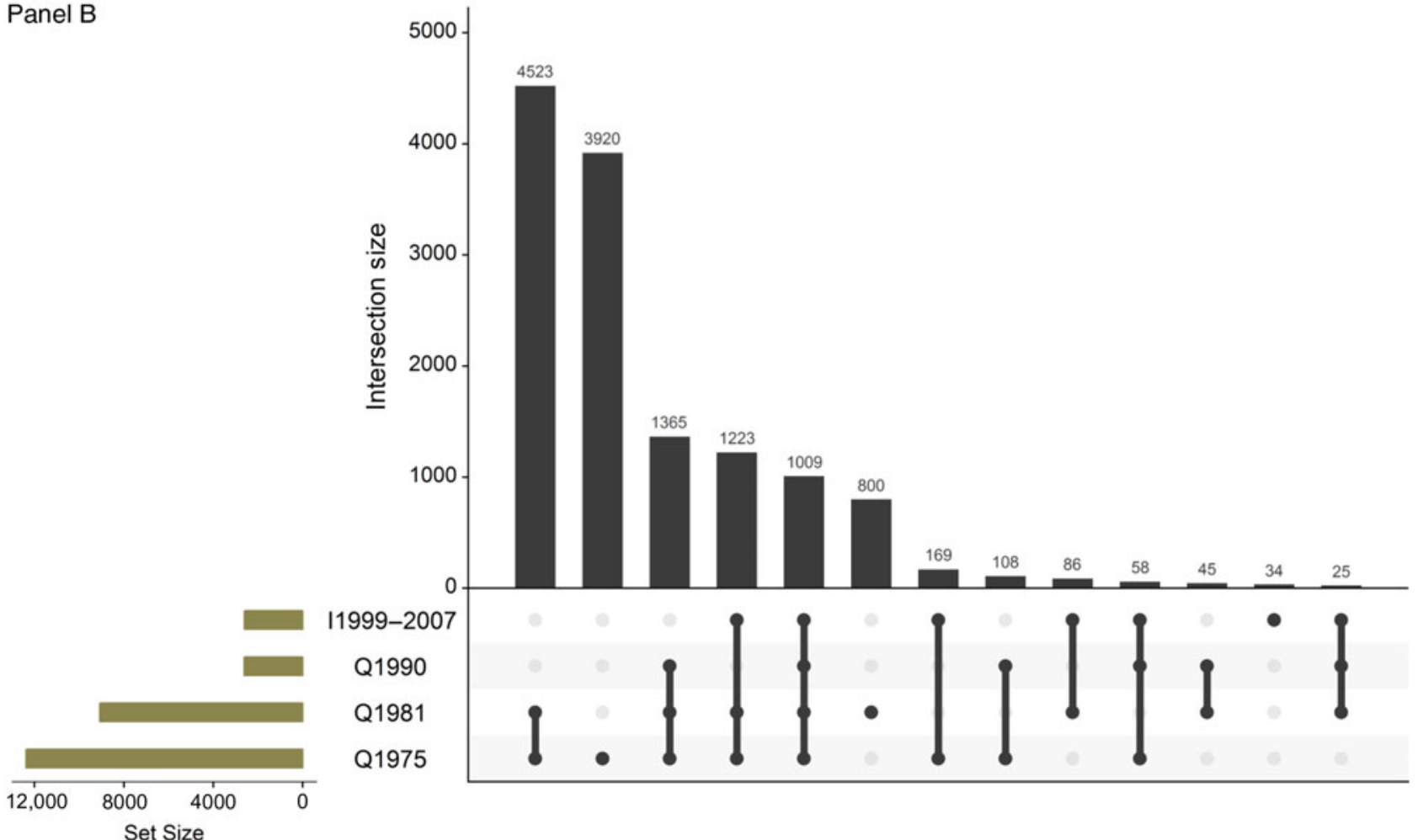

Fig. 1. Overlap in responses to waves 1-4 studies for the FTC. Panel A: The entire cohort; Panel B: twins born before 1938 and assessed for cognition in wave 4 (older part of MEMTWIN); Panel C: twins born 1938-1944 and assessed for cognition in wave 4 (young part of MEMTWIN) and Panel D: twins born in $1945-1957$. 


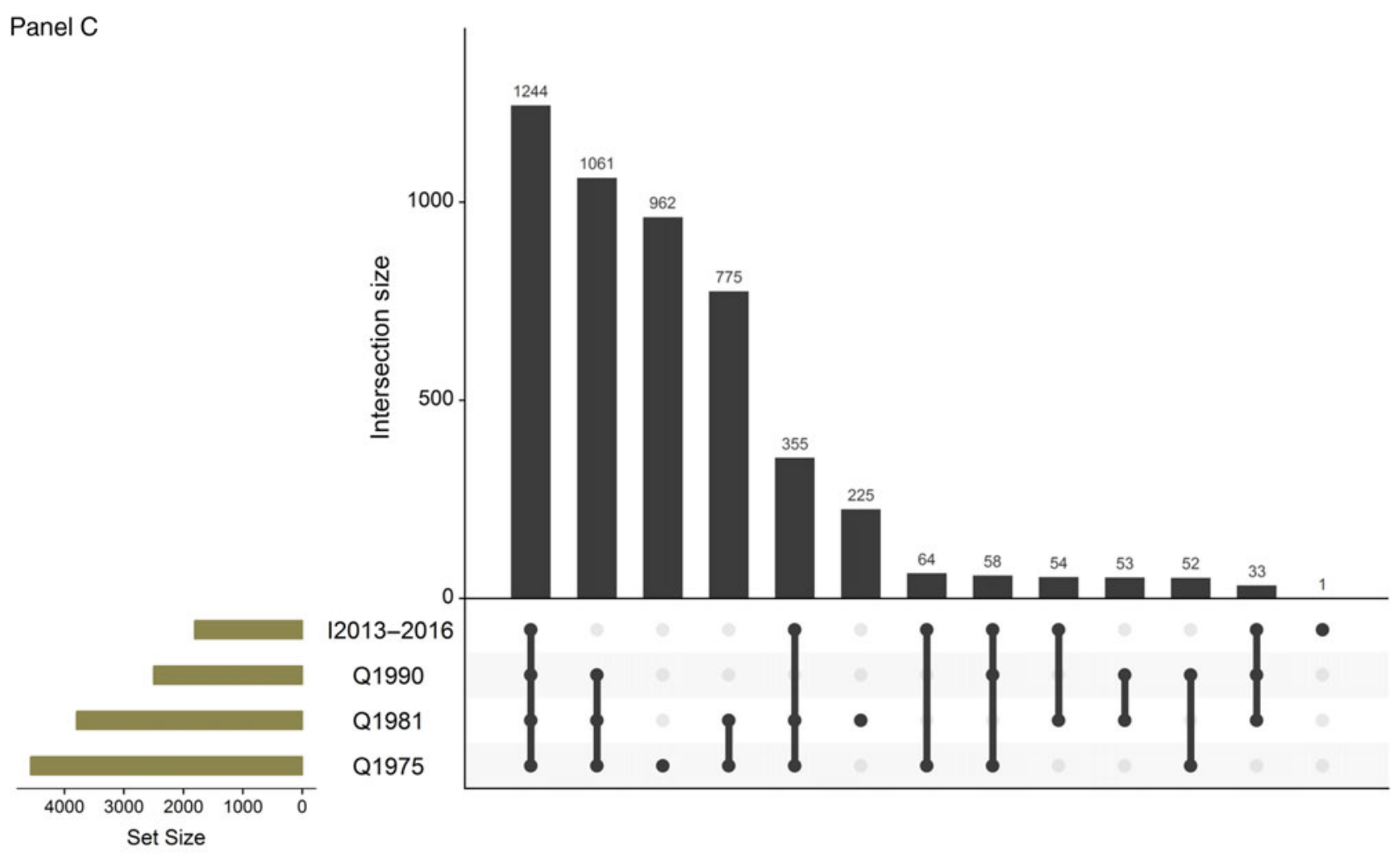

Panel D

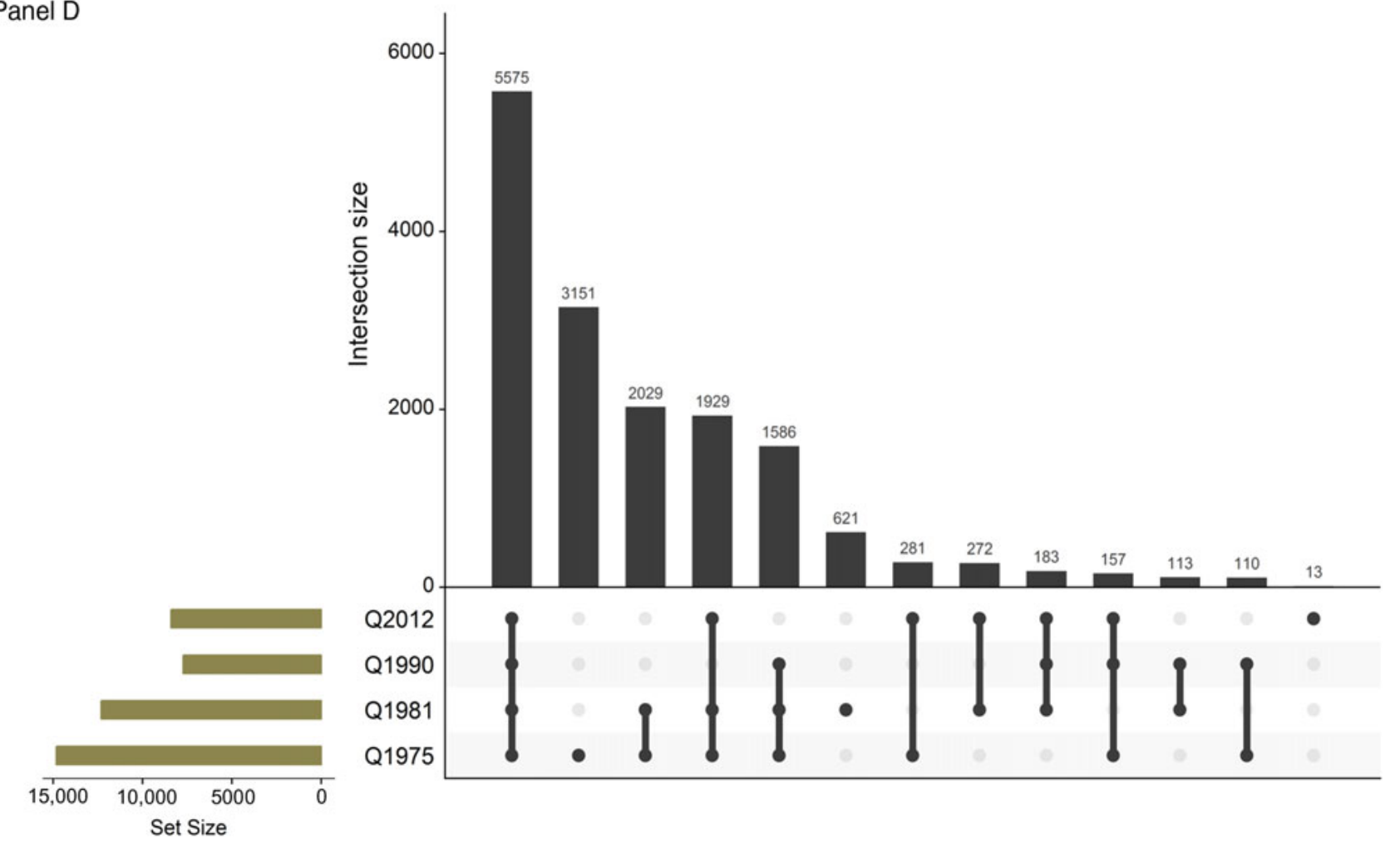

Fig. 1. (continued)

\section{Epigenetics of Hypertension Study}

Based on the 2011 survey, a few years later, we undertook an in-person study on the epigenetics of hypertension (EH-Epi study, see below) as part of a consortium project led by Dr Xiaoling Wang (Georgia Prevention Institute, Augusta University, GA, USA). A total of 447 twins were examined and measured, including four measurements of BP at two separate time points, weight, height as well as waist and hip circumferences, as well as fasting glucose and cell counts (60 items). A lifestyle interview had items on current medications, use of alcohol, smoking, occupation, physical 
Table 2. Number of deaths, cancer cases in the twin cohort by survey year and the numbers genotyped or with methylation data. The number of pairs genotyped on a genomewide array

\begin{tabular}{|c|c|c|c|c|c|c|c|c|c|}
\hline Wave & Mode & Year(s) & Birth years & $\begin{array}{l}N \text { of deaths } \\
\text { by } 1 / 1 / 2018\end{array}$ & $\begin{array}{l}N \text { of cancer } \\
\text { cases by } \\
2016\end{array}$ & $\begin{array}{l}N \text { with } \\
\text { genotype } \\
\text { data }\end{array}$ & $\begin{array}{l}N \text { with } \\
\text { methylation } \\
\text { data }\end{array}$ & $\begin{array}{l}N \text { of MZ pairs with at } \\
\text { least one twin } \\
\text { genotyped }\end{array}$ & $\begin{array}{l}N \text { of DZ pairs with } \\
\text { both twins } \\
\text { genotyped }\end{array}$ \\
\hline Wave 1 & Q & 1975 & -1957 & 12,652 & 5477 & 8579 & 430 & 1427 & 2203 \\
\hline Wave 2 & Q & 1981 & -1957 & 9221 & 5030 & 8404 & 420 & 1404 & 2123 \\
\hline Wave 3 & Q & 1990 & $1930-1957$ & 2530 & 2309 & 6125 & 349 & 961 & 1455 \\
\hline $\begin{array}{l}\text { Wave } 4 \\
\text { (MEMTWIN I) }\end{array}$ & I & $1999-2007$ & Before 1938 & 1413 & 839 & 1952 & 10 & 156 & 437 \\
\hline $\begin{array}{l}\text { Wave } 4 \\
\text { (MEMTWIN II) }\end{array}$ & I & $2003-2016$ & $1938-1944$ & 81 & 334 & 1662 & 2 & 218 & 306 \\
\hline Wave 4 & $Q$ & $2011-2012$ & $1945-1957$ & 375 & 975 & 2743 & 423 & 541 & 600 \\
\hline
\end{tabular}

activity at leisure and handedness (160 items). The participants also completed a 56-item brief food frequency questionnaire.

Blood samples were taken for genetic and epigenetic studies (DNA methylation using 450-k arrays, next-generation sequencing for miRNA and histone modification analyses), as well as for metabolomics on several platforms and transcriptomics. See below for more details and initial results.

\section{Inheritance of Drinking Behavior Cohort}

While the older FTC is large and comprehensive, it is not the first in Finland. There is a well-known study of male twins born in the 1920s who took part in a study of genetics of alcohol use and abuse, summarized in the book Inheritance of Drinking Behavior, by Juha Partanen, Kettil Bruun and Touko Markkanen (Partanen et al., 1966). They studied in detail 902 male twin-pairs aged 28-37 years in 1958, and we have now been able to access this interview data. We are currently re-entering the paper interviews as the previous electronic records no longer exist. The interview was extensive with 182 items on biographical data, relationships between co-twins, external circumstances, drinking behavior, illnesses, food and taste preferences and anthropological measures. In addition, intelligence and personality tests were conducted and blood samples drawn. As many of the pairs have also taken part in our 1975 and 1981 surveys, this dataset will add data from one more data point to the longitudinal study.

\section{Register Linkages}

Through regular register linkages, we have updated vital status and address information as needed, while cancer incidence and cause of death data are updated from the Cancer Registry and Statistics Finland, respectively. For more details on ongoing cancer research, see the review on the Nordic Twin Study of Cancer (Mucci et al., 2016). In addition, some analyses of predictors of cancer incidence have been done on the Finnish data alone. More extensive register linkages have been waiting for new legislation on accessing health and social data. The number of deaths and number of cancer cases (all kinds of malignancies) are shown in Table 2 for the like-sex twins who have replied to the specified surveys. This enables planning of analyses of exposure data collected at the surveys in relation to cause of death or cancer outcomes.

The Finnish Parliament has also approved the Act on the Secondary Use of Health and Social Data in 2019. It enables wider and speedier use of the data not only from national social and health care registers but also from the operational client and patient systems in primary care, specialist health care and social services. Decisions on accessing the data will be taken by the new Data Permit Authority as a centralized national actor. For sensitive data, it will be required to be handled in a safe and secure environment, such that only the results of the analytics can be used externally. Potentially, this will permit linkage of the twin cohort to multiple new registers for novel analyses. The new regulation implements the GDPR (the EU's General Data Protection Regulation) in Finland. It is one of the first implementations of the GDPR for the secondary use of data in Europe.

\section{Biobanking}

As part of the Finnish Biobanking Legislation (law 688/2012), all biological samples associated with the FinnTwin12 study, the FinnTwin16 study and the older FTC have been deposited in 2018 with the nationwide Biobank of the National Institute for Health and Welfare, which has a license to operate from the National Supervisory Authority for Welfare and Health. Biobanked data are publicly available for use by qualified researchers worldwide following a standardized application procedure. Access to coded data is managed and controlled by the Biobank in accordance with the limitations of the consent forms signed by participants and Finnish law. A unique feature of the Biobank is the ability to link study records to Finland's national health and social welfare registries, which includes extensive information regarding medical diagnoses and receipt of social services (e.g., disability claims). For details on accessing the biobanked data on the twins, see https://thl.fi/en/web/thl-biobank/for-researchers/ application-process.

There are also hospital-based biobanks in Finland, where the pathology archives from the past 30 years or more are accessible. We are in the process of linking the older FTC to pathology archives to identify tissue samples from twin-pairs in which one or both twins have selected cancers, such as prostate, breast, colorectal and lung cancer. This has now become easier as there is a single portal, called FINGENIOUS, run by the Finnish Biobank cooperative (https://finbb.fi/), by which one can reach all six Finnish hospital biobanks by completing just one feasibility and access request.

Many DNA samples have been collected during targeted studies of selected pairs, conducted from the early 1990s onward, and all available samples have now been genotyped, imputed and available for genomewide association studies (GWASs) and other analyses. Table 2 also gives numbers of persons with genomewide genotypes 
or methylation data. Please note that the methylation data have generally been collected in later years, and not necessarily at the same time as the latest surveys but rather at the time of the respective substudy during an on-site visit. We also have some information on opposite-sex twins, some of which is register-based (twins born in 1950-1957) and some through questionnaire contact in 1996-1997 for twins born in the 1940s. Across all three cohorts, we have DNA samples on more than 15,000 twins and family members, who have taken part in family studies. Notably, the opposite-sex twins and first-degree relatives in the Nicotine Addiction Genetics (NAG) family study are not tabled here.

Almost 2000 methylation arrays have been done overall with 526 in the old cohort from 442 individuals and 83 with repeated measures. We are currently undertaking more methylation array analyses, which will add some 400 more in the old FTC. The more detailed breakdown of Ns will be given in separate review papers for Finntwin 12 and Finntwin 16 in the forthcoming special issue of twin registries in this journal. In addition, there are transcriptomics, histone modifications, miRNA analyses and metabolomics in selected subsets of twins.

\section{Main Scientific Projects in Recent Years}

In the following sections, the major recent and ongoing scientific projects undertaken based on the FTC are described. The list of projects is not comprehensive, and projects initiated more than 10 years ago that have been completed are not included. Moreover, our contributions of many GWASs and Mendelian randomization summary statistics to multiple consortia exploring the genetics of common, complex traits and conditions are not described in detail.

\section{Hypertension Epigenetics}

Both genetic and environmental factors contribute to the pathogenesis of essential hypertension (EH). The GWASs have shown that the associated single-nucleotide polymorphisms (SNPs) explain less than $6 \%$ of the BP variation in the population (Evangelou et al., 2018; Hoffmann et al., 2017; Wain et al., 2017; Warren et al., 2017). Emerging evidence suggests that epigenetic variants play an important role in the pathogenesis of EH (Kato et al., 2015; Wang et al., 2013). Altogether, 199 twin-pairs and four singletons (age 55-69 years; $60.7 \%$ monozygous) from the $\mathrm{EH}$-Epi substudy were included in epigenome and transcriptomewide analysis of hypertension. We identified 13 novel CpG methylation sites (article in preparation) and one new gene, MOK with its expression associated with BP, and replicated several previously identified signals in both gene expression and DNA methylation level. Results from bivariate twin modeling further provided new insights into the genetic and environmental sources of BP-related DNA methylation and gene expression signatures (Huang et al., 2018).

\section{Smoking and Mental Health Disorders}

Depression and smoking are strongly associated, but the causal nature of their relationship is debated. Depression is a major risk factor for suicide and suicidality, but the role of smoking in suicide is unclear. We have examined longitudinal associations between smoking, depression and suicidality using the data from the older FTC.

We studied smoking behavior in 1975-1981 as a predictor of depression in 1990 (The Beck Depression Inventory, BDI score $>9$ ) among 4164 men and 4934 women. Among the men, persistent smoking and smoking in 1975 but quitting by 1981 were associated with a higher risk of depression, while among the women only the quitters had an elevated risk. When family and genetic background were controlled, smoking remained a predictor of depression. Genetic modeling among men suggested a modest correlation $\left(r_{g}=.25\right)$ between the genetic components of smoking and depression (Korhonen et al., 2007).

We further examined which depression dimensions are linked to smoking patterns (4980 men and 5997 women). Longitudinal cigarette smoking patterns in 1975-1981 included multiple categories describing consistency and change. The three BDI dimensions were negative attitudes toward self (NATS), performance impairment and weight loss assessed in 1990. Persistent smoking and inconsistent former smoking predicted all the depression dimensions, although such associations with the NATS dimension were independent among men only. Long-term abstinence (consistent former smoking) did not predict risk for any depression dimension. Controlling for familial confounding, the association of persistent smoking with later NATS was replicated within discordant twin-pairs, suggesting that smoking is most robustly associated with the 'core dimension' of depression, that is, NATS (Korhonen et al., 2011).

While these previous studies were based on self-reported depressive symptoms, the last study investigated whether smoking predicts register-based outcome, that is, antidepressant medication $(n=10,652)$. A questionnaire was administered to Finnish adult twins in 1990. Antidepressant prescription data during 19952004 were obtained from the register of the Finnish Social Insurance Institution and linked to the survey data. Cox Proportional Hazard Models among 1075 cases and 9577 controls assessed the risk for depression in the cohort, whereas within-pair comparisons of smoking twins with their nonsmoking co-twins controlled for shared familial influences. Based on the analysis among those without baseline depression, heavy daily smokers had a significantly elevated likelihood for antidepressant prescription when adjusted for all confounders. A similar analysis using pairs discordant for antidepressant medication confirmed that daily smoking twins had a higher likelihood for antidepressant prescriptions compared with their nonsmoking co-twins (Korhonen et al., 2017).

Within the older FTC, we investigated the relationship between smoking and suicide among 16,282 twin-pairs born before 1958. Smoking status and dose, marital status, employment and socioeconomic status and indicators of psychiatric and somatic illness were assessed at both 1975 and 1981 surveys. Emergent psychiatric and medical illness and vital status, including suicide determined by forensic autopsy, were evaluated over 35-year follow-up through national registries. The association between smoking and suicide was determined in competing risks hazard models. In twin-pairs discordant for smoking and suicide, the prospective association between smoking and suicide was determined using a matched case-control design. Smokers had a higher cumulative suicide incidence than former or never smokers. Heavy smokers had significantly higher suicide risk than light smokers. Compared with never smokers, smokers, but not former smokers, had increased suicide risk, adjusting for depressive symptoms, alcohol and sedative-hypnotic use, and excluding those who developed a serious somatic or psychiatric illness. In twin-pairs discordant for smoking and suicide, suicide was more likely in smokers. Adults who smoked tobacco were more likely to die by suicide, with a large, dose-dependent effect. This effect remained after consideration of many known predictors of suicide and shared familial effects, consistent with the hypothesis that exposure to tobacco smoke increases the risk of suicide (Evins et al., 2017). 


\section{Sleep}

Sleep is also a major determinant of wellbeing and mental health, and over the past decades we have reported on the genetic and environmental contributions to sleep length and quality, as well as many other sleep-related traits, such as snoring, parasomnias and sleep bruxism. Recently, we have examined longitudinal associations of sleep quality across the four surveys among twins born in 1945-1957, showing that the large majority of respondents sleep well or very well across a 35-year period, while very few are consistently poor sleepers, indicating that more or less short-term circumstances, such as life events or illnesses, probably lie behind episodes of poor sleep, and poor sleep is persistent only among very few individuals (Hublin et al., 2018). A longitudinal analysis of sleep length is ongoing.

\section{Genetics and Epigenetics of Smoking, Nicotine Metabolism and Nicotine Dependence}

Smoking behavior is influenced by both genetic and environmental factors, and epigenetic factors may link these two. FTC twins who have participated in the NAG, Schizophrenia and EH-Epi studies have been used to investigate both genetics and epigenetics and their interaction in smoking-related traits. Data from the FinnTwin12 and FinnTwin16 cohorts have also contributed. Genetics has been assessed by SNPs, while epigenetics has been assessed by measuring DNA methylation using genomewide array technologies.

Using the FinnTwin12 and FinnTwin16 together with other Finnish data $(N=1518)$, we performed the first GWAS on the rate of nicotine metabolism (Loukola et al., 2015), which influences smoking behavior such as response to smoking cessation treatments (Lerman et al., 2006). We found an association locus on chromosome 19, harboring the CYP2A6 gene. Based on our results, we constructed a genetic risk score explaining $~ 30 \%$ of the variance observed in the nicotine metabolite ratio (NMR) to study the association of the NMR and smoking in the older FTC data and other Finnish data. The genetic risk score was positively associated with cigarettes smoked per day (CPD) and smoking cessation, suggesting that individuals with faster nicotine metabolism smoke more, but are more likely to quit. The latter association was no longer statistically significant after accounting for potential confounders, such as quitting due to adverse health consequences or having major depression (Loukola et al., 2015).

Subsequently, we performed GWAS meta-analyses of the NMR and five other smoking-related traits in 5185 Finnish, Canadian and Australian current smokers, including data from the FTC (Buchwald et al., 2019). This was the largest GWAS to date of the NMR, and the first of the nicotine intake biomarker, cotinine +3 hydroxycotinine, and the smoking intensity variable, cotinine/ CPD. We replicated previous findings, such as the chromosome 19 association locus for the NMR, as well as revealed new association loci and shed light on the genetic overlap between the studied traits.

We have also contributed to multiple other meta-analyses of GWAS data on various smoking and alcohol phenotypes, notably the GSCAN consortium GWAS and exome analyses (Brazel et al., 2018; Erzurumluoglu et al., 2019; Liu et al., 2019), for nicotine dependence (Hancock et al., 2018) and alcohol dependence (Walters et al, 2018). These analyses have been based on alcohol and smoking behavior data from the surveys, as well as from the structured psychiatric interviews conducted as part of the NAG study. Additionally, we used the FTC data in our targeted hypothesis-driven study of the neuregulin signaling pathway
(Gupta et al., 2017). Our neuregulin signaling pathway-targeted analyses resulted in identification of SNPs with regulatory potential and provide evidence for the involvement of the pathway in smoking behavior and comorbid diseases. We have also done a GWAS on detailed smoking phenotypes covering the time dependency of progression of a smoker from initiation, to becoming a regular smoker, to smoking cessation based on detailed phenotyping in the NAG study (He et al., 2016).

The epigenome has been screened by epigenomewide association studies to identify genomic regions that are affected by or may influence smoking behavior (Gupta et al., 2019). Genetic and epigenetic data have also been integrated to uncover the regulatory potential of those variants that associate with smoking behavior phenotypes. We have observed multiple cis and trans methylation quantitative trait loci at genomewide significant SNPs or CpG sites and showed that DNA methylation is a molecular mechanism mediating the effects of genotype on smoking behavior phenotypes at some genetic loci (Gupta et al., 2019; Loukola et al., 2015).

As is well known, self-reported smoking status is prone to misclassification due to under-reporting, while biomarkers such as cotinine can measure only recent exposure. It is also known that smoking strongly influences DNA methylation, with current, former and never smokers exhibiting different methylation profiles. To meet the needs for accurate smoking status estimation, we have developed a robust DNA methylation-based predictor, EpiSmokEr, providing an objective measure of smoking status (https://github.com/sailalithabollepalli/EpiSmokEr; Bollepalli et al., 2019). DNA methylation profiles of $405 \mathrm{EH}$-Epi twins were used in this project as a test dataset.

\section{Alcohol Use and Its Consequences Over Time}

Using measures of alcohol use behaviors at each study wave, we investigated birth cohort effects on the quantity and heritability of alcohol use. As expected, twins in earlier birth cohorts were more often abstainers and consumed less alcohol. However, we also found the contribution of genetic and environmental factors to variation in alcohol consumption to vary by birth cohort: in earlier cohorts, environmental factors shared by co-twins were more important, whereas the heritability of alcohol use behaviors was higher in more recent cohorts (Virtanen et al., 2018). The FTC offers unusual opportunities to study long-term associations of alcohol use and test for familial/genetic confounds in comparisons to exposure discordant $\mathrm{MZ}$ twin-pairs. While earlier analyses of alcohol use discordant pairs had not shown differences in mortality, longer follow-up and more refined phenotypes show that even in MZ twin-pairs discordant for alcohol use, the heavier user has a higher risk of death (Sipilä et al., 2015).

We have completed an interview study of twin-pairs discordant for alcohol use in the 1975 baseline. From all pairs born in 1951-1957 in which both co-twins completed the 2011-2012 questionnaire follow-up, 171 pairs, confirmed or assumed to be MZ, were selected for their drinking discordance in 1975 when aged 18-25 years. Questionnaire measures of drinking over the 37-year follow-up confirm substantial stability for both individual differences and intrapair differences for consumption and alcohol problems across the nearly four-decade follow-up. The signed differences in consumption patterns on which pairs were selected in 1975 remained significant (albeit attenuated in magnitude) in the 2011-2012 questionnaire. Semistructured telephone interviews were completed for 128 of these 171 drinking-discordant twin-pairs, and intrapair differences in symptom counts of alcohol use disorder significantly correlated with 
measures of psychological wellbeing, general health, self-esteem and satisfaction with life. A smaller subset of interviewed pairs from the FinnTwin 16 study shows similar findings from earlier ages and over a shorter duration of follow-up; a report of the combined results is in preparation awaiting zygosity confirmation for some twin-pairs.

\section{Leisure-Time Physical Activity, Weight Gain, Health and Mortality - A Prospective Follow-up in Twins}

We have continued the analyses on whether LTPA, adjusted for genetic factors and childhood environment, protects against mortality and different adverse health outcomes. All questionnaires $(1975,1981,1990$ and 2011) included questions on physical activity, allowing for a total metabolic equivalent (MET) index calculation (volume) by multiplying general intensity and the average duration and frequency of physical activity during leisure time. Different Finnish registers were used to study mortality, type 2 diabetes and use of antidepressants (measured as number of purchases). Follow-up telephone interview with detailed questions on the continuation of LTPA, self-measured weight and waist circumference and occurrence of chronic disease was carried out in 2005 (mean age 58.5 years) for physical activity discordant twin-pairs ( $n=146$ twin-pairs).

During follow-up (1976-2004), 1082 type 2 diabetes cases were observed among 20,487 individuals. Among all individuals, participants in MET quintiles (Q) III-V had significantly decreased risk for type 2 diabetes compared with sedentary individuals (QI). The pairwise analyses showed that the body mass index (BMI)-adjusted hazard ratio (HR) for the active (QII-V) compared with sedentary (QI) co-twins at follow-up was decreased by about one-half (0.54) (Waller, Kaprio et al., 2010). Physical activity volume over 15 years (1975-1990) was used as the predictor of subsequent use of antidepressants for 1995-2004 among 642 physical activity discordant twin-pairs (Waller et al., 2016). Altogether, 229 persons had used at least one prescribed antidepressant during the study period. Active co-twins had a $20 \%$ lower risk for using any quantity of antidepressants than their inactive co-twins; trends were similar for DZ (20\% decrease) and MZ pairs (22\% decrease). The lowest odds ratio $(\mathrm{OR})(0.51,0.26-0.98)$ was seen among $\mathrm{MZ}$ pairs after adjusting for BMI, smoking and drinking.

Among the 146 LTPA discordant pairs, 24 co-twins (16 inactive and 8 active) had died between 1981 and 2004. The active cotwins had a strongly reduced risk of all-cause mortality as social class-adjusted HR was significantly reduced ( $\mathrm{HR}=0.39$; Waller, Kujala, Rantanen et al., 2010). This was not found among the small number of MZ pairs. Mortality follow-up was extended for a period of 1981-2013 when twin-pairs discordant for vigorous LTPA were studied (discordance in both 1975 and 1981). This dataset included 1074 activity discordant twin-pairs (of which 231 were MZ pairs). Among 278 activity and death discordant pairs, 180 inactive and 98 active co-twins had died during follow-up. The unadjusted pairwise HR for all pairs was significantly reduced for all pairs (0.64) and for DZ pairs (0.58) but not for MZ pairs (0.85; Karvinen et al., 2015). When this analysis was repeated in the baseline-healthy subgroup, the HRs were further attenuated for DZ pairs and for MZ pairs (Karvinen et al., 2015).

On the other hand, an increased mortality by $41 \%$ was observed among twins with persistent dyspnea (breathlessness) at baseline (dyspnea in 1975 and 1981) compared with asymptomatic individuals. The within-pair analyses of death discordant pairs showed a similar and significant increase of $47 \%$ for all twin-pairs, with a point estimate of $164 \%$ for healthy MZ pairs (Waller et al., 2014).

Among the 95 interviewed physical activity discordant twinpairs, the risk of type 2 diabetes or glucose intolerance $(\mathrm{OR}=0.09, p=.022)$ and incident elevated $\mathrm{BP} \quad(\mathrm{OR}=0.46$, $p=.039)$ was lower among the active co-twins. The active co-twins were more satisfied with their life at follow-up $(p=.047)$. In contrast, the active co-twins showed a tendency toward more sportsrelated injuries $(\mathrm{OR}=1.9, p=.051$; Waller, Kujala, Kaprio et al., 2010). Within the subgroup of 42 pairs discordant for LTPA over 30 years, mean weight gain from 1975 through 2005 was $5.4 \mathrm{~kg}$ less $(p=.003)$ and waist circumference $8.4 \mathrm{~cm}$ smaller $(p<.001)$ at follow-up among the active compared with inactive co-twins (Waller et al., 2008).

A smaller subgroup of twins participated in an accelerometermeasured physical activity study called MOBILETWIN, which is a subset of the MEMTWIN younger twins, as it included all available same-sex twin-pairs born in 1940-1944. Twins completed a telephone interview and answered the questionnaire, and altogether 787 individuals, 404 women (283 complete pairs; 119 MZ pairs, mean age 72.9 years) wore a hip triaxial accelerometer for at least 4 days in 2014-2016.

High baseline mean MET (mean from 1975, 1981 and 1990) index predicted higher amounts of objectively measured moderate-to-vigorous physical activity (MVPA), with an increase in regression model $R^{2}$ of $6.9 \%$ after age- and sex-adjustment $(p<.001)$ at follow-up among individuals (Waller, Vähä-Ypyä, Lindgren et al., 2018). Self-reported fitness at follow-up explained moderately the variation in measured MVPA (age- and sexadjusted $\left.R^{2} 20 \%\right)$. MZ pairs were more similar $(r=.57)$ in objectively measured MVPA at follow-up compared to DZ pairs $(r=.28)$ (Waller, Vähä-Ypyä, Törmäkangas et al., 2018). Using mediation analysis, it was shown that shared genetic factors account for a large share of the associations between midlife and later physical activity variables $\left(r_{\mathrm{G}}=.59 ; 95 \%\right.$ CI $[0.44,0.79]$; Waller, Vähä-Ypyä, Lindgren et al., 2018). In later years, low objectively measured physical activity was associated with the occurrence of chronic diseases (Kujala et al., 2019).

Three sets of analyses have examined causes and consequences of long-term LTPA (>1.5 MET-h/day) and inactivity ( $\leq 1.5 \mathrm{MET}$ $\mathrm{h}$ /day) by comparing 35-year LTPA profiles among twins born in 1945-1957 who replied to all four surveys in 1975, 1981, 1990 and 2011. The associations of 15 -year smoking profile, education attainment by 1981 and 35-year BMI change with physical inactivity were studied (Piirtola et al., 2016; Piirtola, Kaprio, Silventoinen et al., 2017; Piirtola, Kaprio, Waller et al., 2017). We found that long-term persistent daily smoking increased the likelihood of either remaining or becoming physically inactive, whereas quitting smoking decreased this likelihood (Piirtola, Kaprio, Waller et al., 2017). In analyzing long-term smoking discordant twin-pairs, any kind of smoking history was associated with physical inactivity. Further confirmation came from the observation that smoking at midlife also predicted less objective measured MVPA at followup in pairwise analysis among MZ twin-pairs (Waller, Vähä-Ypyä, Törmäkangas et al., 2018). Regarding another major risk factor, we found that higher education protected from physical inactivity because each additional year of education was associated with lower likelihood for inactivity both in the cross-sectional and longitudinal analyses (Piirtola et al., 2016). These associations held true also for within-pair analyses, indicating an independent relationship between education attainment and LTPA. Persistent physical activity was protective against weight gain, also for 
within-pair analyses, though somewhat attenuated coefficients indicating confounding of familiar factors (Piirtola, Kaprio, Waller et al., 2017).

In summary, physical activity helps in maintaining overall health by decreasing the rate of weight gain, lowering waist circumference, reducing the risk for clinical type 2 diabetes and use of antidepressants. However, genetic factors may play a bigger role in explaining the associations between physical activity and mortality, as some of the findings were clearer among the DZ than MZ twin-pairs discordant for LTPA.

\section{Causes and Consequences of Physical Activity: The TWINACTIVE Study}

Sixteen twin-pairs (of whom seven were MZ pairs) with a persistent discordance in their LTPA levels were comprehensively identified from the FTC (Leskinen, Waller et al., 2009). The discordance between the co-twins of the twin-pairs was initially defined from baseline questionnaires (1975 and 1981). A followup interview (from 1980 to 2005) was set to assess discordance in LTPA thereafter. The activity level assessment was based on calculation of the volume of activity, the MET index, from the selfreported physical activity.

Among the 16 pairs, the same twin had higher activity MET index than his or her co-twin throughout the follow-up period up to year 2007 when we conducted the multidimensional health-related measurements. The co-twins did not differ in other lifestyle factors, such as smoking, alcohol or work-related physical activity habits, either at baseline assessment (1975) or at the end of the follow-up (2007). The mean intrapair difference in LTPA MET indexes between the co-twins for the follow-up period was extensive, $8.8 \mathrm{MET} \mathrm{h} /$ day. As a result, the physically active co-twins had higher peak exercise capacity (9.3 METs vs. 7.5 METs, $p<.001$ ) and $20 \%$ higher knee extensor force compared to their inactive co-twins (Leskinen et al., 2009, 2013).

At the end of the follow-up, the inactive twins had a higher overall body fat content, 1.5 times more visceral and extramyocellular thigh fat and a significantly higher liver fat score compared to their active co-twins (Leskinen, Sipilä et al., 2009). These differences were also seen among the $\mathrm{MZ}$ co-twins only (Leskinen \& Kujala, 2015). In skeletal muscle tissue, gene expression of the central pathways of energy metabolism and supportive metabolic pathways, especially those genes related to the processes of oxidative phosphorylation and branched-chain amino acid (BCAA) degradation, were upregulated among the physically active compared to inactive co-twins (Leskinen et al., 2010). In subcutaneous fat tissue samples, the upregulated pathways in active compared to inactive co-twins included BCAA degradation and polyunsaturated fatty acid synthesis.

TWINACTIVE study shows that physically active lifestyle maintains aerobic capacity and muscle strength. Regular physical activity versus inactivity lowers the rate of ectopic fat accumulation even when the genetic background is considered. These findings, among our metabolomics finding (Kujala et al., 2013), indicate lower cardio-metabolic risk among persistently active versus inactive twins.

\section{Predictors and Early Detection of Old Age Cognitive Impairment and Dementia - The MEMTWIN and ADTWIN Studies}

Genetically informed studies of dementia risk factors with assessment of future cognition or dementia are rare; for that reason, the twin studies in our older twins were initiated as described above. On the one hand, questionnaire and register-based information were used (the MEMTWIN study); on the other hand, detailed assessments among selected pairs using neuropsychology and neuroimaging were done (ADTWIN study).

MEMTWIN. Using the whole MEMTWIN data, Paula Iso-Markku (Iso-Markku et al., 2015, 2016, 2018) has built on earlier work by Jyri Virta et al. (2013) to look at physical activity and cognition in particular. Although cross-sectional and longitudinal studies point to an association of physical activity with reduced risk of dementia, no twin studies have so far been able to show a significant association between physical activity and cognition or dementia in a cotwin control design controlling for genetic factors and shared environment. Although the small number of MZ discordant pairs may be part of the explanation, shared genes underlying physical activity and cognition may also account for the lack of association in the within-pair analyses.

By using an older part of the MEMTWIN data, we created a risk score by using only self-reported educational and occupational information (Vuoksimaa et al., 2016). Lower scores - reflecting lower education and lower middle-age occupational status - at a mean age of 47 years predicted cognitive status at a mean age of 74 years with the area under the curve of 0.77 when classifying individuals into cognitively healthy versus demented. The risk score was also significantly associated with cognition in a sample of 1011 individuals from younger birth cohorts (born in 19381944) forming the MEMTWIN data where the mean follow-up time was 39 years. Not only years of education were the strongest predictor of old age cognition but also four questions about middle-age occupational status had an independent effect on old age cognition (Vuoksimaa et al., 2016).

Using the entire MEMTWIN data, we investigated the heritability and the effect of having a cognitively impaired co-twin on episodic memory performance in 4367 twins, including 553 MZ, $823 \mathrm{DZ}$ and 19 unknown zygosity pairs (Lindgren et al., 2019). Immediate free recall of 10 -word list (single trial) had a heritability estimate of 0.37 with the remaining variance explained by unique environmental effects. Looking at twin-pairs discordant for their TELE performance (normal vs. impaired cognition), after adjusting for age, education and sex, cognitively healthy twins recalled more words (3.79) than their demented co-twins (3.05). Moreover, cognitively healthy twins with demented co-twins recalled fewer words than twins from cognitively healthy pairs (4.23; Lindgren et al., 2019). These findings show that familial risk of dementia is evident in poorer episodic memory performance even in the presence of normative score in a cognitive screening instrument. This highlights the importance of using more specific measures of episodic memory in the early detection of cognitive impairment. These results also suggest that immediate free recall measure may be useful endophenotype for genetic studies of dementia.

ADTWIN - Brain imaging of twins discordant for cognitive status. Based on MEMTWIN data collection, we have invited twin-pairs discordant for cognitive status to participate in the ADTWIN study with a laboratory protocol including NP testing and brain imaging (Scheinin et al., 2011; Virta et al., 2009). The NP test battery included the Consortium to Establish a Registry for Alzheimer's Disease Neuropsychological Battery, subtests from the Wechsler Memory Scale - Revised and the Wechsler Adult Intelligence Scale - Revised, as well as other NP tests. 
Cognitive discordance was based on telephone interview and verified with the NP tests.

Brain imaging included MRI and PET with variable tracers. In cohorts $<1938$, PET was used to measure $\beta$-amyloid accumulation with ${ }^{11} \mathrm{C}$-Pittsburgh compound B (PiB) PET and cerebral glucose metabolism with ${ }^{18} \mathrm{~F}$-Fluorodeoxyglucose (FDG) PET. Both tracers were also used to study cognitively healthy nontwin controls. The study using $\mathrm{PiB}$ included 17 cognitively discordant twin-pairs (Scheinin et al., 2011). Those with cognitive impairment had $\mathrm{PiB}$ uptake patterns typical to Alzheimer's disease. Moreover, cognitively healthy MZ co-twins had higher $\beta$-amyloid deposition, whereas no difference in $\beta$-amyloid levels was evident in healthy co-twins from DZ pairs compared to controls. In MZ pairs, cognitively preserved co-twins showed similar $\mathrm{PiB}$ uptake patterns as their cognitively impaired co-twins suggesting that genetic factors influence $\beta$-amyloid pathology (Scheinin et al., 2011).

FDG study included 16 twin-pairs and showed that cerebral glucose metabolism was lower in the demented co-twins compared to controls. The nondemented MZ co-twins showed reduced glucose metabolism, whereas no reductions were found in the nondemented DZ co-twins. The lower cerebral glucose metabolism in nondemented MZ twins with a demented co-twin may indicate genetic susceptibility to Alzheimer's disease (Virta et al., 2009).

In the younger screened cohorts born in 1938-1944, PET imaging was done in 2014-2017 and a total of 17 twin-pairs participated in 11C-PiB PET and 11 twin-pairs participated in 11C-PRB28 PET (measuring neuroinflammation marker translocator protein) imaging. These discordant twin analyses are currently ongoing.

\section{Future Prospects - Cognitive Aging in the Oldest-Old}

Given that the older FTC included all Finnish same-sex twin-pairs born before 1958, many of these individuals have reached the age of 90 years. People who are 90 years or older are commonly referred to as the oldest-old, and they are the fastest growing population segment in many countries, including Finland. A big challenge is that we know very little about cognitive and brain aging in the oldest-old. Still, the prevalence and incidence of dementia are highest in this age group (Brumback-Peltz et al., 2011). On the other hand, some of the oldest-old maintain their cognitive functioning and do have good memory even when compared to younger age groups.

The effects of risk factors of dementia may differ as a function of age. In addition to low education attainment, middle-age cardiovascular risk factors are the most robust risk factors of dementia. However, it is not clear whether the risk factor-dementia associations are similar across old age. For example, hypertension with onset after 80 years of age was related to decreased risk of dementia in the oldest-old (Corrada et al., 2017). Likewise, metabolic syndrome, a cluster of risk factors (hypertension, obesity, dyslipidemia and impaired glucose metabolism) of cardiovascular disease, has been reported to be associated with attenuated cognitive decline in the oldest-old (van den Berg et al., 2007). Many studies in the oldest-old have a short follow-up time, which may lead to reverse causation when investigating the risk factors after the disease process has started. Results based on several cohorts, including the older FTC, suggest that higher BMI is associated with higher dementia risk when the follow-up is over 20 years, whereas no association is observed with a follow-up of 10-20 years and there is an inverse association between BMI and dementia with less than
10 years of follow-up (Kivimäki et al., 2018). Genetic risk of dementia may also differ as a function of age: having an $A P O E$ $€ 4$-allele confers risk for $\mathrm{AD}$ in people $\geq 65$ years, but this effect seems to be attenuated among the oldest-old (Corrada et al., 2013; Valerio et al., 2014). Taken together, the middle-age factors that predict dementia or successful cognitive aging in the oldestold are not well known. Survival bias in studies of $\geq 90$ year olds may result in having participants with high levels of resilience/cognitive reserve, as indexed by protective factors such as higher general cognitive ability and higher education or protective genetic background.

To learn more about the risk factors for dementia in the oldestold, we are going to administer the telephone interview protocol to collect cognitive data from the FTC participants who are 90 years or older. Every year, more than 100 individuals are expected to reach the age of 90 years. These data together with the questionnaire data from 1975 will allow investigating the longitudinal association with a follow-up time of 45 years or more. Moreover, we can utilize the longitudinal FTC questionnaire data from the 1981 and 1990 questionnaires. Establishing a new database in 90-year-old twins will also make it possible to conduct more indepth laboratory assessment, for example, by using the ADTWIN protocol of studying twin-pairs discordant for cognitive functioning. Also, registry-based data in the FTC can be used to study whether the risk factor-dementia associations are similar across the old age.

\section{Finnish Twin Study on Aging}

The purpose of the Finnish Twin Study on Aging (FITSA) is to study the genetic and environmental effects on the functional phenotypes that are relevant for the disablement process among older women. As FITSA includes three study waves, it is a longitudinal study embedded within the larger cohort.

The FITSA participants were recruited from the older FTC, including 1260 female twin-pairs born in 1924-1937 (Kaprio \& Koskenvuo, 2002; Kaprio et al., 1978; Sarna et al., 1978). Of those women who were 63-76 years old in 2000, and who had participated in the FTC surveys in 1975 and 1981, invitations to take part in the FITSA were sent to 414 female twin-pairs living in Finland and $103 \mathrm{MZ}$ and $114 \mathrm{DZ}$ twin-pairs participated. The day-long protocol included the following assessments: biosampling, clinical measurements, anthropometrics, body composition, physical performance and sensory functions. Information about health habits and outcomes such as functional abilities and disabilities were collected in a questionnaire. Surveillance data on falls were collected for 1 year after the laboratory examination using a calendar method. Mortality has been followed up from the national registers.

First follow-up measurements of FITSA were conducted after 3 years, in 2003-2004. Altogether, 419 women participated in the follow-up study, of whom 313 participated in the laboratory measurements and filled in a questionnaire, while 106 women responded solely to the postal questionnaire. The second followup round for FITSA took place in 2011-2012. Altogether, 344 (91\%) of the 377 surviving participants answered a structured, postal questionnaire, which was supplemented with telephone interviews when needed.

There are multiple dissertations and publications about FITSA pheno- and genotypes; for example, according to quantitative genetic analyses, muscle strength, power and walking speed have 
a genetic effect in common (Tiainen et al., 2007). Studies about falls have revealed that familial factors underlie the risk of falling (Pajala et al., 2006) and that falls, as well as fear of falling, are associated with vision and hearing acuity (Kulmala et al., 2009; Viljanen et al., 2009, 2013). Hearing and vision are both largely accounted for by genetic differences between individuals (Pärssinen et al., 2013, 2015, 2016, 2019; Viljanen, Era et al., 2007; Viljanen, Kaprio et al., 2007). The FITSA study has contributed new knowledge especially in the area of genetic and environmental effects on phenotypes relevant for sensory and physical functioning underlying the disablement process in old age.

Because FITSA participants have also taken part in the FTC surveys in years 1975 and 1981, it has been possible to do longitudinal analyses in a genetically controlled sample with over 35 -year follow-up from persons' middle age to old age. It has been discovered, for example, that extraverted personality at middle age protected from, while neuroticism increased, the risk of later life depressive symptoms (Pakkala et al., 2010). Middle-age living habits had no effect on depressive symptoms at old age. According to genetic analyses, neuroticism at middle age and depressive symptoms at old age shared an additive genetic component, while such a common genetic component was not observed with extraversion (Pakkala et al., 2010). We have also reported that during the 29-year follow-up, BMI increased by $17 \%$. Midlife BMI was a significant predictor of mobility 29 years later. Genetic influences on BMI level and its rate of change accounted for $37 \%$ and $25 \%$, respectively, of the genetic influences on mobility later in life. The corresponding environmental influences on BMI level and its rate of change were $35 \%$ and $22 \%$ (Ortega-Alonso et al., 2009). FITSA data have also been utilized in several genetic studies. For example, vision, hearing and forced vital capacity have been analyzed as a part of larger multisite consortia identifying several new associated loci/genes (Loth et al., 2014; Vuckovic et al., 2015).

\section{Estrogen and Sarcopenia: A Co-Twin Study With HRT Discordant Postmenopausal Women (The SAWES Study)}

Hormonal aging has notable effects on the functions of different organs and body systems as well as on functional capacity and health among middle-aged and older people. The most notable and wellcharacterized age-induced change in hormonal systems is the decline in sex hormone production in women during the menopausal transition. Low sex hormone concentrations are among the possible key mechanisms for sarcopenia. Sarcopenia is characterized by progressive loss of skeletal muscle mass and strength with an increased risk of adverse outcomes such as physical disability, poor health and poor quality of life and death. The underlying biological mechanisms by which age-induced sex hormone deprivation affects muscle mass and function are largely unknown.

The Sarcopenia - Skeletal Muscle Adaptation to Postmenopausal Hypogonadism and Effects of Hormone Replacement Therapy and Physical Activity in Older Women: a Genetic and Molecular Biological Study on Estrogen-related Pathways (SAWEs) study was launched in 2007 with the aim of identifying the key genetic factors needed for maintaining proper muscle mass, composition and function after menopause and to understand their connection with estrogen status and physical activity.

An invitation to participate the SAWEs study was sent to all female MZ twin-pairs born in 1943-1952 (537 pairs). Of these, 114 pairs responded and 21 pairs identified themselves as being
HRT discordant, that is, one sister was a current HRT user, while the other had never used HRT. Finally, after confirming zygosity, $15 \mathrm{MZ}$ twin-pairs from those who met the inclusion criteria formed the study group. At time of the study, enrolled participants were 54-62 years old and their HRT discordance ranged from 2 to 16 years, with a mean (SD) duration of 6.9 (4.1) years. Of the users, five women used estradiol preparations, six used estradiol with progestogen preparation and four women used tibolone.

This co-twin control study found postmenopausal HRT use to be beneficial for skeletal muscle. In particular, the greater relative area of the thigh muscles and the lower relative fat area within the muscle compartment among the sisters on HRT than their sisters with no history of HRT were found (Ronkainen et al., 2009). No significant differences between the sisters were found in maximal voluntary isometric force (Finni et al., 2011; Ronkainen et al., 2009). However, when the effect of the central nervous system (voluntary activation) was blocked using electrical stimulation to the peripheral nerve, the force output of the plantar flexors was 32\% greater among the HRT users compared to nonusers (Finni et al., 2011). Accordingly, the specific tension of single type I and IIa myofibres was significantly greater among the HRT users than nonusers (Qaisar et al., 2013). In fast and more complex voluntary neuromuscular functions, such as the vertical countermovement jump and maximal walking speed, the sisters on HRT performed better than the nonusing sisters (Ronkainen et al., 2009).

Estrogens have been suggested to play a role in tendon abnormalities, injuries and tendon diameter. Achilles tendon thickness and cross-sectional area did not significantly differ between HRT using and nonusing twin sisters. However, in five physically active pairs, the sister receiving HRT had a smaller tendon crosssectional area than their sisters (Finni et al., 2009). In order to investigate the influence of long-term HRT use on bone strength and its determinants, the peripheral computed tomography data on tibial shaft, distal tibia and distal radius acquired from the HRT discordant twin-pairs at the FITSA (9 pairs) and SAWEs (15 pairs) studies were combined (Mikkola et al., 2011). The study found no difference between HRT users and nonusers in the total bone cross-sectional area in any of the measured bone sites but instead showed HRT users to have higher bending strength of the tibial shaft to be accounted from the larger cortical area and higher cortical bone mineral density. Furthermore, the estimated compressive strength of the distal tibia and distal radius was also higher among the HRT users compared to their nonusing co-twins.

Several approaches have been taken in the SAWEs to investigate the underlying molecular mechanisms. The investigation of the global muscle gene expression pointed toward estrogenic regulation of cell structure, cell-matrix interactions, energy metabolism and utilization of nutrients pathways (Ronkainen et al., 2010). Further studies showed that the whole-body level differences favoring lower adiposity, higher lean mass and better muscle performance among HRT users were also apparent in the adiposity markers measured from blood circulation (Ahtiainen et al., 2012) and in the pathways identified from muscle proteomic study (Laakkonen et al., 2017). The focused inspection of the intramuscular sex steroid hormones (including estradiol, testosterone, dihydrotestosterone and dehydroepiandrosterone) revealed each hormone to be an independent predictor of female muscle strength and power regardless of age, systemic estradiol and body adiposity (Pöllänen et al., 2015).

Several microRNA-related studies have also been conducted using samples from SAWEs. MicroRNAs are small regulatory 
RNA molecules that function as repressors of the protein synthesis and thereby regulate the activity of multitude of biological processes. MicroRNAs may directly affect the cells they have been produced but they can also be transported to the blood circulation and function as endocrine regulatory elements. Within the SAWEs study, we were first to identify estradiol-responsive microRNAs from skeletal muscle (Olivieri et al., 2014) and later expanded these studies to include adipose tissue (Kangas, Morsiani et al., 2017) and circulating microRNAs (Kangas et al., 2014; Kangas, Törmäkangas et al., 2017). The muscle-expressed estradiolresponding microRNAs, miR-182, miR-223 and miR-142-3p, had common targets in the insulin/IGF-1 pathway, which is known to participate in the regulation of muscle mass (Olivieri et al., 2014). The circulating miRs showing differential expression due to use of HRT were associated with apoptosis, inflammation and body metabolic pathways (Kangas et al., 2014; Kangas, Morsiani et al., 2017). These results are in line with our previous results indicating that the use of HRT affects muscle signaling at transcriptome (Ronkainen et al., 2010) and proteome (Laakkonen et al., 2017) levels, as well as white blood cell signaling at methylome and transcriptome levels (Bahl et al., 2015).

Taken together, results from the SAWEs study indicate that menopausal changes in circulating steroid hormones, especially in female sex steroid estradiol, have multilevel effects on skeletal muscle gene expression, which can be seen at the phenotype level as increased adiposity, decreased lean mass, poorer bone properties and reduced muscle performance. The use of HRT partially aids maintaining better body composition and muscle function.

\section{Biological Aging Clocks and Physical Activity and Functioning}

Biological clocks, of which the most promising have been telomere length and DNA methylation age, also known as the 'Epigenetic clock', present an exciting opportunity to track and help understand the human biological aging process (Horvath, 2013; Jylhävä et al., 2017). We have investigated genetic and environmental predictors of telomere length and DNA methylation age, and how these clocks are associated with not only determinants of older age physical functioning and LTPA at the total body level but also in muscle tissue, which has a vital role for health and functioning in old age. The aim of these studies has been to increase knowledge about the biological mechanisms underlying the development of age-related functional limitations and diseases.

Telomere length measurements by quantitative polymerase chain reaction (PCR) have been performed at baseline to subsample FITSA $(n=386)$. For SAWEs twins, telomere length measurements have also been conducted from muscle samples with the Southern blot method (Sillanpää, Niskala et al., 2017). We collaborated in a large European meta-analysis, which showed that telomere length is related to lung function and lung diseases (Albrecht et al., 2014). Following that, we showed that the associations between telomere length and lung phenotypes are explained by shared genetic factors underlying both phenotypes (Sillanpää, Sipilä et al., 2017). Our analysis also revealed that telomere length predicts a decline in physical functioning over an 11-year followup (Sillanpää et al., 2016) and that telomere length is causally associated with cognitive functions (Hägg et al., 2017). These results indicate that telomeres are genetically regulated biomarkers that can be used to predict the development of disability on older age. However, as predictors of life or health span, their value is rather low and overall, associations between telomeres and physical functioning are weak. Telomeres measured from muscle tissue may be more closely related to, for example, age-related loss in muscle mass and strength than telomeres measured from white blood cells (Sillanpää, Niskala et al., 2017).

Recently, we have expanded our biological aging research into DNA methylation clocks. DNA methylation age estimates can be produced from data generated by DNA methylation arrays. Currently, about 2000 DNA samples from individuals in the twin cohorts have been analyzed by Illumina HumanMethylation $450 \mathrm{k}$ or EPIC BeadChip. In comparison to telomeres, DNA methylation age may be more closely associated with physical functioning phenotypes in older people (Sillanpää et al., 2018). Quantitative genetic modeling in older and younger FTC revealed that the relative contribution of nonshared environmental factors was larger among older compared with younger twin-pairs (Sillanpää et al., 2019). We tested the hypothesis that long-term LTPA is one of the environmental factors that affect variation in DNAm age in older age. A co-twin control analysis with older same-sex twinpairs who had persistent discordance in physical activity for 32 years according to reported/interviewed physical-activity data (TWINACTIVE) showed no differences in DNA methylation age among active and inactive co-twins (Sillanpää et al., 2019). The nonexistent or at most very minor associations between LTPA and DNA methylation age were confirmed also in crosssectional analysis in a sample of FinnTwin16 twin-pairs.

\section{International Activities}

The FTC data, from all three cohorts (this one, FinnTwin12 and FinnTwin16) and our research group, have been active in multiple international consortia. There have been multiple consortia for specific molecular genetic analyses, where the primary goal has been to increase prediction accuracy and to achieve deeper insight into complex trait biology by studying sample sizes as large as possible. For these, we have conducted GWAS analyses locally and shared summary statistics for the meta-analysis. Local knowledge of the phenotype, the circumstances of data collection and the characteristics of the genotyping array are essential to make a successful contribution. Likewise, we have contributed to epigenomewide association study meta-analyses and Mendelian randomization meta-analyses. Only a few of the conducted analyses are mentioned above.

Two more ambitious and long-term collaborations have been the Nordic Twin study on Cancer and the CODAtwins study, where we have been very active with leadership roles. Both international collaborations are reviewed in the forthcoming special issue on twin registries. We have also contributed to other international collaborations, including IGEMS described in the special issue. While we are open to collaboration, the current European data protection legislation, which is very recently updated, places constrictions on our ability to share person-level data from the entire cohort surveys and interviews, as the limits of what is and what is not possible are still not clear at the moment. Please contact the first author for more information. On the other hand, access to the data in the biobank - that is, the twins and family members with biosamples and appropriate consents provides novel opportunities for researchers worldwide.

Financial support. The support for data collection and analyses of the papers reviewed in the manuscript are mentioned in the individual articles. Support for the preparation of this manuscript has been provided by the Academy of Finland (grants 312073 to J. Kaprio, 314639 and 320109 to E. Vuoksimaa, 
310526 to T. Rantanen, 308698 to A. Latvala and 297908 to M. Ollikainen). N. Lindgren was supported by the Päivikki and Sakari Sohlberg Foundation. SA was supported by the Finnish Ministry of Education and Culture, the Juho Vainio Foundation and the Finnish Cultural Foundation. T. Rantanen has been supported by the European Research Council (grant number 693045) and R. J. Rose by AA- 012502

\section{Conflicts of interest. None.}

\section{References}

Ahtiainen, M., Alén, M., Pöllänen, E., Pulkkinen, S., Ronkainen, P. H. A., Kujala, U. M., ... Kovanen, V. (2012). Hormone therapy is associated with better body composition and adipokine/glucose profiles: A study with monozygotic co-twin control design. Menopause, 19, 1329-1335.

Albrecht, E., Sillanpää, E., Karrasch, S., Alves, A. C., Codd, V., Hovatta, I., \& Schulz, H. (2014). Telomere length in circulating leukocytes is associated with lung function and disease. European Respiratory Journal, 43, 983-992.

Bahl, A., Pöllänen, E., Ismail, K., Sipilä, S., Mikkola, T. M., Berglund, E., . . . Ollikainen, M. (2015). Hormone replacement therapy associated white blood cell DNA methylation and gene expression are associated with within-pair differences of body adiposity and bone mass. Twin Research and Human Genetics, 18, 647-661.

Bollepalli, S., Korhonen, T., Kaprio, J., Ollikainen, M., \& Anders, S. (2019). EpiSmokEr: A robust classifier to determine smoking status from DNA methylation data. bioRxiv, 487975.

Brandt, J., Spencer, M., \& Folstein, M. (1988). The telephone interview for cognitive status. Neuropsychiatry, Neuropsychology \& Behavioral Neurology, 1, 111-117.

Brazel, D. M., Jiang, Y., Hughey, J. M., Turcot, V., Zhan, X., Gong, J., . . . Vrieze, S. (2018). Exome chip meta-analysis fine maps causal variants and elucidates the genetic architecture of rare coding variants in smoking and alcohol use. Biological Psychiatry, 85, 946-955.

Brumback-Peltz, C., Balasubramanian, A. B., Corrada, M. M., \& Kawas, C. H. (2011). Diagnosing dementia in the oldest-old. Maturitas, 70, 164-168.

Buchwald, J., Chenoweth, M. J. Palviainen, T., Zhu, G., Benner, C., Gordon, S., ... Tyndale, R. F. (2019). Genome-wide association meta-analysis of nicotine metabolism and cigarette consumption measures in smokers of European descent. Manuscript submitted for publication.

Corrada, M. M., Hayden, K. M., Paganini-Hill, A., Bullain, S. S., DeMoss, J., Aguirre, C., .. Kawas, C. H. (2017). Age of onset of hypertension and risk of dementia in the oldest-old: The 90+ study. Alzheimer's \& Dementia, 13, 103-110.

Corrada, M. M., Paganini-Hill, A., Berlau, D. J., \& Kawas, C. H. (2013). Apolipoprotein E genotype, dementia, and mortality in the oldest old: The 90+ study. Alzheimer's \& Dementia: The Journal of the Alzheimer's Association, 9, 12-18.

Erzurumluoglu, A. M., Liu, M., Jackson, V. E., Barnes, D. R., Datta, G., Melbourne, C., ... Howson, J. M. M. (2019). A meta-analysis of up to 622,409 individuals identifies 40 novel smoking behaviour associated genetic loci. Molecular Psychiatry. Advance online publication. https://doi.org/10. 1038/s41380-018-0313-0

Evangelou, E., Warren, H. R., Mosen-Ansorena, D., Mifsud, B., Pazoki, R., Gao, H., ... Caulfield, M. J. (2018). Genetic analysis of over 1 million people identifies 535 new loci associated with blood pressure traits. Nature Genetics, 50, 1412-1425.

Evins, E., Korhonen, T., Kinnunen, T. H., \& Kaprio, J. (2017). Prospective association between tobacco smoking and death by suicide: A competing risks hazard analysis in a large twin cohort with 35-year follow up. Psychological Medicine, 47, 2143-2154.

Finni, T., Kovanen, V., Ronkainen, P. H., Pöllänen, E., Bashford, G. R., Kaprio, J., ... Sipilä, S. (2009). Combination of hormone replacement therapy and high physical activity is associated with differences in Achilles tendon size in monozygotic female twin pairs. Journal of Applied Physiology (1985), 106, 1332-1337.

Finni, T., Noorkoiv, M., Pöllänen, E., Ronkainen, P. H., Alén, M., Kaprio, J., .. Sipilä, S. (2011). Muscle function in monozygotic female twin pairs discordant for hormone replacement therapy. Muscle Nerve, 44, 769-775.
Gatz, M., Reynolds, C. A., John, R., Johansson, B., Mortimer, J. A., \& Pedersen, N. L. (2002). Telephone screening to identify potential dementia cases in a population-based sample of older adults. International Psychogeriatrics, 14, 273-289.

Gupta, R., Qaiser, B., He, L., Hiekkalinna, T. S., Zheutlin, A. B., Therman, S., . Loukola, A. (2017). Neuregulin signaling pathway in smoking behavior. Translational Psychiatry, 7, e1212.

Gupta, R., van Dongen, J., Fu, Y., Abdellaoui, A., Tyndale, R. F., Velagapudi, V., Ollikainen, M. (2019). Epigenome-wide association study of serum cotinine in current smokers reveals novel genetically driven loci. Clinical Epigenetics, 11, 1.

Hägg, S., Zhan, Y., Karlsson, R., Gerritsen, L., Ploner, A., van der Lee, S. J., Pedersen, N. L. (2017). Short telomere length is associated with impaired cognitive performance in European ancestry cohorts. Translational Psychiatry, 7, e1100.

Hancock, D. B., Guo, Y., Reginsson, G. W., Gaddis, N. C., Lutz, S. M., Sherva, R., Johnson, E. O. (2018). Genome-wide association study across European and African American ancestries identifies a SNP in DNMT3B contributing to nicotine dependence. Molecular Psychiatry, 23, 1911-1919.

He, L., Pitkäniemi, J., Heikkilä, K., Chou, Y. L., Madden, P. A., Korhonen, T., ... Loukola, A. (2016). Genome-wide time-to-event analysis on smoking progression stages in a family-based study. Brain and Behavior, 6, e00462.

Hoffmann, T. J., Ehret, G. B., Nandakumar, P., Ranatunga, D., Schaefer, C., Kwok, P. Y., ... Risch, N. (2017). Genome-wide association analyses using electronic health records identify new loci influencing blood pressure variation. Nature Genetics, 49, 54-64.

Horvath, S. (2013). DNA methylation age of human tissues and cell types. Genome Biology, 14, R115.

Huang, Y., Ollikainen, M., Sipilä, P., Mustelin, L., Wang, X., Su, S., .. . Wang, X. (2018). Genetic and environmental effects on gene expression signatures of blood pressure: A transcriptome-wide twin study. Hypertension, 71, 457-464.

Hublin, C., Lehtovirta, M., Partinen, M., Koskenvuo, M., \& Kaprio, J. (2018). Changes in sleep quality with age - A 36-year follow-up study of Finnish working-aged adults. Journal of Sleep Research, 27, e12623.

Iso-Markku, P., Waller, K., Kujala, U. M., \& Kaprio, J. (2015). Physical activity and dementia: Long-term follow-up study of adult twins. Annals of Medicine, 47, 81-87.

Iso-Markku, P., Waller, K., Vuoksimaa, E., Heikkilä, K., Rinne, J., Kaprio, J., \& Kujala, U. M. (2016). Midlife physical activity and cognition later in life: A prospective twin study. Journal of Alzheimers Disease, 54, 1303-1317.

Iso-Markku, P., Waller, K., Vuoksimaa, E., Vähä-Ypyä, H., Lindgren, N., Heikkilä, K., . . Kujala, U. M. (2018). Objectively measured physical activity profile and cognition in Finnish elderly twins. Alzheimers \& Dementia (NY), 4, 263-271.

Jylhävä, J., Pedersen, N. L., \& Hägg, S. (2017). Biological age predictors. EBioMedicine, 21, 29-36.

Kangas, R., Morsiani, C., Pizza, G., Lanzarini, C., Aukee, P., Kaprio, J., . . . Capri, M. (2017). Menopause and adipose tissue: miR-19a-3p is sensitive to hormonal replacement. Oncotarget, 9, 2279-2294.

Kangas, R., Pöllänen, E., Rippo, M. R., Lanzarini, C., Prattichizzo, F., Niskala, P., ... Kovanen, V. (2014). Circulating miR-21, miR-146a and Fas ligand respond to postmenopausal hormone replacement therapy A study with monozygotic twin pairs. Mechanisms of Ageing and Development, 15, 1-8.

Kangas, R., Törmäkangas, T., Fey, V., Pursiheimo, J., Miinalainen, I., Alen, M. . Laakkonen, E. K. (2017). Aging and serum exomiR content in women Effects of estrogenic hormone replacement therapy. Scientific Reports, 14, 42702.

Kaprio, J. (2006). Twin studies in Finland 2006. Twin Research and Human Genetics, 9, 772-777.

Kaprio, J. (2013). The Finnish Twin Cohort Study: An update. Twin Research and Human Genetics, 16, 157-162.

Kaprio, J., \& Koskenvuo, M. (2002). Genetic and environmental factors in complex diseases: The older Finnish Twin Cohort. Twin Research, 5, 358-365.

Kaprio, J., Sarna, S., Koskenvuo, M., \& Rantasalo, I. (1978) The Finnish twin registry: Formation and compilation, questionnaire study, zygosity 
determination procedures, and research program. Progress in Clinical and Biological Research, 24, 179-184.

Karvinen, S., Waller, K., Silvennoinen, M., Koch, L. G., Britton, S. L., Kaprio, J., Kujala, U. M. (2015). Physical activity in adulthood: Genes and mortality. Scientific Reports, 5, Article no. 18259.

Kato, N., Loh, M., Takeuchi, F., Verweij, N., Wang, X., Zhang, W., .. Chambers, J. C. (2015). Trans-ancestry genome-wide association study identifies 12 genetic loci influencing blood pressure and implicates a role for DNA methylation. Nature Genetics, 47, 1282-1293.

Kivimäki, M., Luukkonen, R., Batty, G. D., Nyberg, S. T., Alfredsson, L., Fransson, E. I., ... Jokela, M. (2018). Body mass index and risk of dementia: Analysis of individual-level data from 1.3 million individuals. Alzheimers \& Dementia, 14, 601-609.

Korhonen, T., Broms, U., Varjonen, J., Romanov, K., Koskenvuo, M., Kinnunen, T., \& Kaprio, J. (2007). Smoking behaviour as a predictor for depression among Finnish men and women - A prospective study of adult twins. Psychological Medicine, 37, 705-715.

Korhonen, T., Koivumaa-Honkanen, H., Varjonen, J., Broms, U., Koskenvuo, M., \& Kaprio, J. (2011). Cigarette smoking and dimensions of depressive symptoms: Longitudinal analysis among Finnish male and female twins. Nicotine \& Tobacco Research, 13, 261-272.

Korhonen, T., Ranjit, A., Tuulio-Henriksson, A., \& Kaprio, J. (2017). Smoking status as a predictor of antidepressant medication use. Journal of Affective Disorders, 207, 221-227.

Kujala, U. M., Hautasaari, P., Vähä-Ypyä, H., Waller, K., Lindgren, N., Iso-Markku, P., ... Sievänen, H. (2019). Chronic diseases and objectively measured physical activity among aged individuals - A cross-sectional twin cohort study. Annals of Medicine, 51, 78-87.

Kujala, U. M., Mäkinen, V.-P., Heinonen, I., Soininen, P., Kangas, A. J., Leskinen, T., ... Ala-Korpela, M. (2013). Long-term leisure-time physical activity and serum metabolome. Circulation, 127, 340-348.

Kulmala, J., Viljanen, A., Sipilä, S., Pajala, S., Pärssinen, O., Kauppinen, M., Rantanen, T. (2009). Poor vision accompanied with other sensory impairments as a predictor of falls in older women. Age and Ageing, 38, 162-167.

Laakkonen, E. K., Soliymani, R., Karvinen, S., Rintala, P., Kaprio, J., Kujala, U., ... Lalowski, M. (2017). Estrogenic regulation of skeletal muscle proteome: A study of premenopausal women and postmenopausal MZ cotwins discordant for hormonal therapy. Aging Cell, 16, 1276-1287.

Lerman, C., Tyndale, R., Patterson, F., Wileyto, E. P., Shields, P. G., Pinto, A., \& Benowitz, N. (2006) Nicotine metabolite ratio predicts efficacy of transdermal nicotine for smoking cessation. Clinical Pharmacology \& Therapeutics, 79, 600-608.

Leskinen, T., \& Kujala, U. M. (2015). Health-related findings among twin pairs discordant for leisure-time physical activity for 32 years: The TWINACTIVE study synopsis. Twin Research and Human Genetics, 18, 266-272.

Leskinen, T., Rinnankoski-Tuikka, R., Rintala, M., Seppänen-Laakso, T., Pöllänen, E., Alen, M., . . Kujala, U. M. (2010). Differences in muscle and adipose tissue gene expression and cardio-metabolic risk factors in the members of physical activity discordant twin pairs. Plos One, 5, e12609.

Leskinen, T., Sipilä, S., Alen, M., Cheng, S., Pietiläinen, K. H., Usenius, J.-P., Kujala, U. M. (2009). Leisure time physical activity and high-risk fat: A longitudinal population-based twin study. International Journal of Obesity, 33, 1211-1218.

Leskinen, T., Sipilä, S., Kaprio, J., Kainulainen, H., Alen, M., \& Kujala, U. M. (2013). Physically active lifestyle, muscle properties and glucose homeostasis in middle-aged and older twins. Age, 35, 1917-1926.

Leskinen, T., Waller, K., Mutikainen, S., Aaltonen, S., Ronkainen, P. H. A., Alen, M., ... Kujala, U. M. (2009). Effects of 30-year leisure time physical activity discordance in twin pairs on health (TWINACTIVE study); aims, design and results for physical fitness. Twin Research and Human Genetics, 12, 108-117.

Lindgren, N., Kaprio, J., Rinne, J. O., \& Vuoksimaa, E. (2019). Immediate verbal recall and familial dementia risk: Population-based study of over 4000 twins. Journal of Neurology, Neurosurgery \& Psychiatry, 90, 90-97.

Liu, M., Jiang, Y., Wedow, R., Li, Y., Brazel, D. M., Chen, F., . . Vrieze, S. (2019). Association studies of up to 1.2 million individuals yield new insights into the genetic etiology of tobacco and alcohol use. Nature Genetics, 51, 237-244.
Loth, D., Artigas, M., Gharib, S., Wain, L. V., Franceschini, N., Koch, B., . . London, S. J. (2014). Genome-wide association analysis identifies six new loci associated with forced vital capacity. Nature Genetics, 46, 669-677.

Loukola, A., Buchwald, J., Gupta, R., Palviainen, T., Hällfors, J., Tikkanen, E., \& Kaprio, J. (2015). Genome-wide association study of a biomarker of nicotine metabolism. PLOS Genetics, 11, e1005498.

Mikkola, T. M., Heinonen, A., Kovanen, V., Cheng, S., Kujala, U. M., Suominen, H., . . Sipilä, S. (2011). Influence of long-term postmenopausal hormone-replacement therapy on estimated structural bone strength: A study in discordant monozygotic twins. Journal of Bone and Mineral Research, 226, 546-552.

Mucci, L. A., Hjelmborg, J. B., Harris, J. R., Czene, K., Havelick, D. J., Scheike, T., ... Nordic Twin Study of Cancer (NorTwinCan) Collaboration. (2016). Familial risk and heritability of cancer among twins in Nordic countries. JAMA, 315, 68-76.

Olivieri, F., Ahtiainen, M., Lazzarini, R., Pöllänen, E., Capri, M., Lorenzi, M., \& Procopio, A. D. (2014). Hormone replacement therapy enhances IGF-1 signaling in skeletal muscle by diminishing miR-182 and miR-223 expressions: A study on postmenopausal monozygotic twin pairs. Aging Cell, 13, 850-61

Ortega-Alonso, A., Sipilä, S., Kujala, U. M., Kaprio, J., \& Rantanen, T. (2009). Genetic influences on adult body mass index followed over 29 years and their effects on late-life mobility: A study of twin sisters. Journal of Epidemiology \& Community Health, 63, 651-658.

Pajala, S., Era, P., Koskenvuo, M., Kaprio, J., Viljanen, A., \& Rantanen, T. (2006). Genetic factors and susceptibility to falls in older women. Journal of the American Geriatrics Society, 54, 613-618.

Pakkala, I., Read, S., Kaprio, J., Koskenvuo, M., Kauppinen, M., \& Rantanen, T. (2010). Genetic contribution to the relationship between personality and depressive symptoms among older women. Psychological Medicine, 40, $1357-1366$.

Pärssinen, O., Kauppinen, M., Halekoh, U., Kaprio, J., \& Rantanen, T. (2019). Heredity of interocular similarities in components of refraction: A population-based twin study among 66- to 79-year-old female twins. Acta Ophthalmologica. Advance online publication. doi: 10.1111/aos.14033

Pärssinen, O., Kauppinen, M., Kaprio, J., Koskenvuo, M., \& Rantanen, T. (2013). Heritability of refractive astigmatism: A population-based twin study among 63- to 75-year-old female twins. Investigative Ophthalmology \& Visual Science, 54, 6063-6067.

Pärssinen, O., Kauppinen, M., Kaprio, J., Koskenvuo, M., \& Rantanen, T. (2015). Heritability of anterior chamber depth and axial length: A population-based twin study among 66 to 79 -year old female twins. Acta Ophthalmologica, 93, e177-178.

Pärssinen, O., Kauppinen, M., Kaprio, J., \& Rantanen, T. (2016). Anisometropia of ocular refractive and biometric measures among 66- to 79-year-old female twins. Acta Ophthalmologica, 94, 768-774.

Partanen, J., Bruun, K., \& Markkanen, T. (1966). Inheritance of drinking behavior, Study No. 14. Helsinki, Finland: The Finnish Foundation for Alcohol Studies.

Piirtola, M., Kaprio, J., Kujala, U. M., Heikkilä, K., Koskenvuo, M., Svedberg, P., ... Ropponen, A. (2016). Association between education and future leisure-time physical inactivity: A study of Finnish twins over a 35-year follow-up. BMC Public Health, 16, 720.

Piirtola, M., Kaprio, J., Silventoinen, K., Svedberg, P., Korhonen, T., \& Ropponen, A. (2017). Association between long-term smoking and leisure-time physical inactivity: A cohort study among Finnish twins with a 35-year follow-up. International Journal of Public Health, 62, 819-829.

Piirtola, M., Kaprio, J., Waller, K., Heikkilä, K., Koskenvuo, M., Svedberg, P., Ropponen, A. (2017). Leisure-time physical inactivity and association with body mass index: A Finnish Twin Study with a 35-year follow-up. International Journal of Epidemiology, 46, 116-127.

Pöllänen, E., Kangas, R., Horttanainen, M., Niskala, P., Kaprio, J., Butler-Browne, G., ... Kovanen, V. (2015). Intramuscular sex steroid hormone are associated with skeletal muscle strength and power in women with different hormonal status. Aging Cell, 14, 236-248.

Qaisar, R., Renaud, G., Hedstrom, Y., Pöllänen, E., Ronkainen, P., Kaprio, J., . Larsson, L. (2013). Hormone replacement therapy improves contractile function and myonuclear organization of single muscle fibres from 
postmenopausal monozygotic female twin pairs. Journal of Physiology, 591, 2333-2344.

Radloff, L. S. (1977). The CES-D Scale: A self-report depression scale for research in the general population. Applied Psychological Measures, 1, 385-401.

Ronkainen, P. H. A., Kovanen, V., Alén, M., Pöllänen, E., Palonen, E. M., Ankarberg-Lindgren, C., ... Sipilä, S. (2009). Postmenopausal hormone replacement therapy modifies skeletal muscle composition and function: A study with monozygotic twin pairs. Journal of Applied Physiology, 107, 25-33.

Ronkainen, P. H. A., Pöllänen, E., Alén, M., Pitkänen, R., Puolakka, J., Kujala, U. M., ... Kovanen, V. (2010). Global gene expression profiles in skeletal muscle of monozygotic female twins discordant for hormone replacement therapy. Aging Cell, 9, 1098-1110.

Sarna, S., Kaprio, J., Sistonen, P., \& Koskenvuo, M. (1978). Diagnosis of twin zygosity by mailed questionnaire. Human Heredity, 28, 241-254.

Scheinin, N. M., Aalto, S., Kaprio, J., Koskenvuo, M., Räihä, I., Rokka, J., . . Rinne, J. O. (2011). Early detection of Alzheimer disease: ${ }^{11} \mathrm{C}-\mathrm{PiB}$ PET in twins discordant for cognitive impairment. Neurology, 77, 453-460.

Sillanpää, E., Kaprio, J., Laakkonen, E. K., Vaara, E., Rantanen, T., Kovanen, V., . Ollikainen, M. (2018). Biological clocks and physical functioning in monozygotic female twins. BMC Geriatrics, 18, 83.

Sillanpää, E., Niskala, P., Laakkonen, E. K., Ponsot, E., Alén, M., Kaprio, J., . . . Sipilä, S. (2017). Leukocyte and skeletal muscle telomere length and body composition in monozygotic twin pairs discordant for long-term hormone replacement therapy. Twin Research and Human Genetics, 20, 119-131.

Sillanpää, E., Ollikainen, M., Kaprio, J., Wang, X., Leskinen, T., Kujala, U. M., \& Törmäkangas, T. (2019). Leisure-time physical activity and DNA methylation age - A twin study. Clinical Epigenetics, 11, 12.

Sillanpää, E., Sipilä, S., Törmäkangas, T., Kaprio, J., \& Rantanen, T. (2017). Genetic and environmental effects on telomere length and lung function: A twin study. The Journals of Gerontology. Series A, Biological sciences and Medical Sciences, 73, 1561-1568.

Sillanpää, E., Törmäkangas, T., Rantanen, T., Kaprio, J., \& Sipilä, S. (2016). Does telomere length predict decline in physical functioning in older twin sisters during 11-year follow-up? Age (Dordrecht), 38, 1-10.

Sipilä, P., Rose, R. J., \& Kaprio, J. (2015). Drinking and mortality: Long-term follow-up of drinking-discordant twin pairs. Addiction, 111, 245-254.

Tiainen, K., Pajala, S., Sipilä, S., Kaprio, J., Koskenvuo, M., Alén, M., . . . Rantanen, T. (2007). Genetic effects in common on maximal walking speed and muscle performance in older women. Scandinavian Journal of Medicine and Science in Sports, 17, 274-280.

Tuomela, J., Kaprio, J., Sipilä, P. N., Silventoinen, K., Wang, X., Ollikainen, M., \& Piirtola, M. (in press). Accuracy of self-reported anthropometric measures Findings from the Finnish Twin Study. Obesity Research and Clinical Practice.

Valerio, D., Raventos, H., Schmeidler, J., Beeri, M. S., Villalobos, L. M., Bolanos-Palmieri, P., ... Silverman, J. M. (2014). Association of apolipoprotein E-e4 and dementia declines with age. The American Journal of Geriatric Psychiatry, 22, 957-960.

van den Berg, E., Biessels, G. J., de Craen, A. J., Gussekloo, J., \& Westendorp, R. G. (2007). The metabolic syndrome is associated with decelerated cognitive decline in the oldest old. Neurology, 69, 979-985.

Viljanen, A., Era, P., Kaprio, J., Pyykkö, I., Koskenvuo, M., \& Rantanen, T. (2007). Genetic and environmental influences on hearing in older women. The Journals of Gerontology, Series A: Biological Sciences and Medical Sciences, 62A, 447-452.

Viljanen, A., Kaprio, J., Pyykkö, I., Sorri, M., Kauppinen, M., Koskenvuo, M., \& Rantanen, T. (2007). Genetic and environmental influences on hearing at different frequencies separately for the better and worse hearing ear in older women. International Journal of Audiology, 46, 772-779.

Viljanen, A., Kaprio, J., Pyykkö, I., Sorri, M., Pajala, S., Kauppinen, M., . . Rantanen, T. (2009). Hearing as a predictor of falls and postural balance in older female twins. The Journals of Gerontology, Series A: Biological Sciences and Medical Sciences, 64A, 312-317.
Viljanen, A., Kulmala, J., Rantakokko, M., Koskenvuo, M., Kaprio, J., \& Rantanen, T. (2013). Accumulation of sensory difficulties predicts fear of falling in older women. Journal of Aging and Health, 25, 776-791.

Virta, J. J., Aalto, S., Järvenpää, T., Karrasch, M., Kaprio, J., Koskenvuo, M., . . Rinne, J. O. (2009). Voxel-based analysis of cerebral glucose metabolism in mono- and dizygotic twins discordant for Alzheimer disease. Journal of Neurology, Neurosurgery \& Psychiatry, 80, 259-266.

Virta, J. J., Heikkila, K., Perola, M., Koskenvuo, M., Raiha, I., Rinne, J. O., \& Kaprio, J. (2013). Midlife cardiovascular risk factors and late cognitive impairment. European Journal of Epidemiology, 28, 405-416.

Virtanen, S., Kaprio, J., Viken, R., Rose, R., \& Latvala, A. (2018). Birth cohort effects on the quantity and heritability of alcohol consumption in adulthood: A Finnish longitudinal twin study. Addiction, 114, 836-846.

Vuckovic, D., Dawson, S., Scheffer, D.I, Rantanen, T., Morgan, A., Di Stazio, M, .. Girotto, G. (2015). Genome-wide association analysis on normal hearing function identifies PCDH20 and SLC28A3 as candidates for hearing function and loss. Human Molecular Genetics, 24, 5655-5664.

Vuoksimaa, E., Rinne, J. O., Lindgren, N., Heikkilä, K., Koskenvuo, M., \& Kaprio, J. (2016). Middle age self-report risk score predicts cognitive functioning and dementia in 20-40 years. Alzheimers and Dementia (Amsterdam), 4, 118-125.

Wain, L. V., Vaez, A., Jansen, R., Joehanes, R., van der Most, P. J., Erzurumluoglu, A. M., ... Ehret, G. B. (2017). Novel blood pressure locus and gene discovery using genome-wide association study and expression data sets from blood and the kidney. Hypertension, 70, e4-e19.

Waller, K., Kaprio, J., Korhonen, T., Tuulio-Henriksson, A., \& Kujala, U. M. (2016). Persistent leisure-time physical activity in adulthood and use of antidepressants: A follow-up study among twins. Journal of Affective Disorders, 200, 172-177.

Waller, K., Kaprio, J., \& Kujala, U. M. (2008). Associations between long-term physical activity, waist circumference and weight gain: A 30-year longitudinal twin study. International Journal of Obesity, 32, 353-361.

Waller, K., Kaprio, J., \& Kujala, U. M. (2014). Dyspnea and all-cause mortality: 28-year follow-up study among adult twins. Medicine \& Science in Sports \& Exercise, 46, 1538-1545.

Waller, K., Kaprio, J., Lehtovirta, M., Silventoinen, K., Koskenvuo, M., \& Kujala, U. M. (2010). Leisure-time physical activity and type 2 diabetes during a 28-year follow-up in twins. Diabetologia, 53, 2531-2537.

Waller, K., Kujala, U. M., Kaprio, J., Koskenvuo, M., \& Rantanen, T. (2010). Effect of physical activity on health in twins: A 30-year longitudinal study. Medicine \& Science in Sports \& Exercise, 42, 658-664.

Waller, K., Kujala, U. M., Rantanen, T., Kauppinen, M., Silventoinen, K., Koskenvuo, M., \& Kaprio, J. (2010). Physical activity, morbidity and mortality in twins: A 24-year prospective follow-up. European Journal of Epidemiology, 25, 731-739.

Waller, K., Vähä-Ypyä, H., Lindgren, N., Kaprio, J., Sievänen, H., \& Kujala, U. M. (2018). Self-reported fitness and objectively measured physical activity profile among older adults: A twin study. The Journals of Gerontology, Series $A$, gly263. Advance online publication.

Waller, K., Vähä-Ypyä, H., Törmäkangas, T., Hautasaari, P., Lindgren, N., Iso-Markku, P., \& Kujala, U. M. (2018). Long-term leisure-time physical activity and other health habits as predictors of objectively monitored late-life physical activity - A 40-year twin study. Scientific Reports, 8, 9400

Walters, R. K., Polimanti, R., Johnson, E. C., McClintick, J. N., Adams, M. J., Adkins, A. E., ... Agrawal, A. (2018). Transancestral GWAS of alcohol dependence reveals common genetic underpinnings with psychiatric disorders. Nature Neuroscience, 21, 1656-1669.

Wang, X., Falkner, B., Zhu, H., Shi, H., Su, S., Xu, X., . . Snieder, H. (2013). A genome-wide methylation study on essential hypertension in young African American males. PLoS One, 8, e53938.

Warren, H. R., Evangelou, E., Cabrera, C. P., Gao, H., Ren, M., Mifsud, B., Wain, L. V. (2017). Genome-wide association analysis identifies novel blood pressure loci and offers biological insights into cardiovascular risk. Nature Genetics, 49, 403-415. 